\title{
Generation of nitric oxide by olive (Olea europaea L.) pollen during in vitro germination and assessment of the S-nitroso- and nitro- pollen proteomes by computational predictive methods
}

\author{
María José Jimenez-Quesada ${ }^{1}$, Rosario Carmona ${ }^{1}$, Elena Lima-Cabello ${ }^{1}$, José Ángel Traverso ${ }^{1, \S}$, \\ Antonio Jesús Castroo ${ }^{1}$ M. Gonzalo Claros ${ }^{2}$, Juan de Dios Alché ${ }^{*}$ \\ ${ }^{1}$ Plant Reproductive Biology Laboratory, Department of Biochemistry, Cellular and Molecular Biology \\ of Plants, Estación Experimental del Zaidín (CSIC), Profesor Albareda 1, 18008 Granada, Spain \\ ${ }^{2}$ Departamento de Biología Molecular y Bioquímica, Universidad de Málaga, Málaga, Spain \\ ${ }^{\S}$ Present address: Department of Cell Biology, Faculty of Science, Granada University, Granada, Spain \\ *To whom correspondence should be addressed. E-mail juandedios.alche@eez.csic.es
}

\begin{abstract}
Nitric oxide is recognized as a signaling molecule involved in a broad range of physiological processes in plants including sexual reproduction. NO has been detected in the pollen grain at high levels and regulates pollen tube growth. Previous studies demonstrated that NO as well as ROS are produced in the olive reproductive tissues in a stage- and tissue-specific manner. The aim of this study was to assess the production of NO throughout the germination of olive (Olea europaea L.) pollen in vitro. The NO fluorescent probe DAF-2DA was used to image NO production in situ, which was correlated to pollen viability. Moreover, by means of a fluorimetric assay we showed that growing pollen tubes release NO. GSNO - a mobile reservoir of NO, formed by the $S$-nitrosylation of NO with reduced glutathione (GSH) was for the first time detected and quantified at different stages of pollen tube growth using a LC-ES/MS analysis. Exogenous NO donors inhibited both pollen germination and pollen tube growth and these effects were partially reverted by the specific NO-scavenger c-PTIO. However, little is known about how NO affects the germination process. With the aim of elucidating the putative relevance of protein $S$ nitrosylation and Tyr-nitration as important post-translational modifications in the development and physiology of the olive pollen, a de novo assembled and annotated reproductive transcriptome from olive was challenged in silico for the putative capability of transcripts to become potentially modified by $S$ nitrosylation/Tyr-nitration according to well-established criteria. Numerous gene products with these characteristics were identified, and a broad discussion as regards to their potential role in plant reproduction was built after their functional classification. Moreover, the importance of both $S$ nitrosylation/Tyr-nitrations was experimentally assessed and validated by using Western blotting, immunoprecipitation and proteomic approaches.
\end{abstract}

\section{KEYWORDS}

Germination, olive, pollen, $S$-nitrosylation, transcriptome, Tyr-nitration 


\section{INTRODUCTION}

Nitric oxide (NO) is an important signaling molecule in physiological and pathological processes in all living beings. It represents a gaseous, relatively high reactive free radical, soluble in aqueous solution while able to diffuse through biological membranes [1].

In mammals, the sources of NO are NO synthase (NOS) enzymes, which catalyze the oxidation of arginine to NO and citrulline. However, plant NO origin is more complex, as NOS-like activity has been described although the gene encoding the enzyme did not. In fact, up to seven pathways for NO biosynthesis have been proposed in plants and this multiplicity difficult clarifying the route for NO production (reviewed in $[2 ; 3 ; 4])$.

In plants, NO has a central role in the wide range of mechanisms involved in growth and development as well as in plant immunity, the hypersensitive response and abiotic interactions (reviewed in [3]). Apparently contradictory functions are exerted by NO (i.e. in plant defence $v s$ symbiotic interaction), and such functions seem to depend on NO concentration and location [4].

NO exerts its role by modulating gene expression or interacting with second messengers, protein kinases, phytohormones or ion channels $[5 ; 6 ; 7]$. NO interaction with ROS has been previously reported [8; 9; 10]. Numerous post-translational modifications (PTMs) are NO-dependent, including cysteine $S$ nitrosylation, tyrosine nitration and metal nitrosylation [6;11]. $S$-nitrosylation is a major PTM involved in plant cell signaling, which takes place when NO reversibly reacts with reduced thiols of a cysteinyl residue (Cys) forming $S$-nitrosothiols (RSNOs). This NO binding to a Cysteine residue affects proteins modulating their activity [12]. $S$-nitrosylation is a reversible PTM and this way can act as an on/off switch control of cell signaling [12]. There are several mechanisms for protein $S$-nitrosylation in biological system, being trans-nitrosylation the most common [13]. Thus, a key reaction takes place between NO and glutathione (GSH), forming $S$-nitrosoglutathione (GSNO), which can be considered a mobile reservoir capable of mediate nitrosylation reactions [14;15]. GSNO level is controlled by the enzyme GSNO-reductase (GSNOR) [2]. On the other hand, protein denitrosylation may be achieved with the involvement of GSNOR and thioredoxin, as well as through non-enzymatic ways [13]. $S$-nitrosylated proteins identified in different species belong to groups as diverse as metabolic enzymes, stress-related proteins, cytoskeletal, photosynthesis, redox-related, and signaling proteins, indicating a broad functional spectrum.

Protein tyrosine nitration $\left(\mathrm{NO}_{2}-\mathrm{Tyr}\right)$ is an alternative PTM mediated by the peroxynitrite $\left(\mathrm{ONOO}^{-}\right)$ generated through the reaction of nitric oxide with superoxide $\left(\mathrm{O}_{2}{ }^{-}\right)$. This PTM affects protein function in most cases irreversibly, and contributes to protein regulation, cellular metabolism and signaling pathways mediated by NO $[6 ; 11 ; 16 ; 17]$.

$\mathrm{NO}$ and ROS have been proposed as signaling mechanism during the development of the plant reproductive tissues, pollen-pistil interaction, pollen tube guidance and double fertilization [5]. At the onset of the progamic phase, initially dehydrated pollen grains adhered to the stigma begin to produce NO 
on a large scale. For its part, receptive stigmas from different angiosperms accumulate $\mathrm{ROS} / \mathrm{H}_{2} \mathrm{O}_{2}[18 ; 19$; $20 ; 21 ; 22 ; 23 ; 24]$. During self-incompatibility response in Papaver rhoeas, a $\mathrm{Ca}^{2+}$-dependent increase in NO and ROS is upstream of programmed cell death (PCD) [25], showing a distinctive reticulate NOpattern. Incompatible olive pollen is the subject of PCD mechanisms, which were suggested to be triggered by the peroxynitrite generated through the reaction of $\mathrm{NO}$ and superoxide [26].

NO donors have been shown to delay pollen germination and tube growth in a dose-dependent manner in different species of angiosperms such as Camellia sinensis, Cucumis sativus or lily, whereas NO scavenger or NOS inhibitor exert the opposite effect [10;27; 28]. On the other hand, gymnosperms pollen seems to behave in the opposite way [19].

NO produced during pollen tube growth reaches a peak in the subapical domain -where peroxisomes are located- with very low labeling in the tip of lily or Pinus bungeana pollen tubes. Such a low abundance of NO at the tip may be a prerequisite for pollen tube growth [18; 19]. Furthermore, NO could act as a chemotropic molecule involved in proper pollen tube guidance to the ovule [18]. Interaction of NO with $\mathrm{Ca}^{2+}$, which has a well-known role in polar cell expansion, was also demonstrated in pollen tubes [18; 19; 20].

Most mentioned studies have been carried out so far in a few model species including Arabidopsis or Lilium, although it could be hypothesized that NO involvement in sexual plant reproduction is a conserved feature among species. To test this hypothesis, we tried to advance in the knowledge of NO functions in the reproductive biology of an evolutionarily distant plant like the olive tree, a woody plant of paramount agronomical interest. Thus, we focused on NO implications in key reproductive parameters such as olive pollen viability, pollen ability to germinate and pollen tube elongation. Furthermore, we wanted to determine whether NO was released into the medium as it occurs with GSNO and with many proteins involved in pollen-stigma interaction. With the aim of studying how NO affects pollen germination and tube elongation, we also envisaged the use of NO donors and scavengers. Finally, we wanted to investigate how NO could exert its regulatory function in pollen through the potential generation of NO-dependent PTMs like S-nitrosylation and Tyr-nitration. This last subject was dealt with throughout an extensive in silico analysis, which also was complemented with an initial experimental validation.

\section{MATERIALS AND METHODS}

\section{Plant material and growth conditions.}

Olea europaea L. plant material was collected from selected olive trees of the cultivar 'Picual', located at the Estación Experimental del Zaidín (CSIC, Granada, Spain). Olive pollen samples were collected during the anthesis in large paper bags by vigorously shaking the inflorescences, and were sequentially sieved through 150 and $50 \mu \mathrm{m}$ mesh nylon filters to eliminate debris. All biological samples were immediately used or stored at $-80^{\circ} \mathrm{C}$. 
In vitro olive pollen germination was initiated by a pre-hydration step in a humid chamber at room temperature for $30 \mathrm{~min}$. Pollen was then re-suspended in a complex germination medium [10\% (w/v) sucrose, $0.03 \%(\mathrm{w} / \mathrm{v}) \mathrm{Ca}\left(\mathrm{NO}_{3}\right)_{2}, 0.01 \%(\mathrm{w} / \mathrm{v}) \mathrm{KNO}_{3}, 0.02 \%(\mathrm{w} / \mathrm{v}) \mathrm{MgSO}_{4}, 0.03 \%(\mathrm{w} / \mathrm{v}$ ) boric acid and 5 $\%(w / v)$ PEG8000], and cultured as described previously [29].

\section{Treatments with NO donors and scavengers}

Stock solutions of $100 \mathrm{mM}$ sodium nitroprusside (SNP, Fluka), $100 \mathrm{mM}$ GSNO (Sigma-Aldrich) and 50 mM 2-(4-carboxyphenyl)-4,4,5,5-tetramethylimidazoline-1-oxyl-3-oxide (c-PTIO, $\mathrm{K}^{+}$salt, Calbiochem) were prepared in deionized water immediately before use. Nitric oxide donors (SNP and GSNO) were used at different concentrations $(0,10,50,100,500$ and $1000 \mu \mathrm{M})$, while the NO scavenger c-PTIO was used at a final dilution of $200 \mu \mathrm{M}$. To study the effects of NO donors and scavengers on pollen germination, these chemicals were added to the medium at the onset of the culture. To analyze their effects on pollen tube elongation, SNP, GSNO and c-PTIO were added to the culture medium once pollen tubes started to emerge, and pollen tubes were left to grow for five additional hours.

\section{Cellular detection of NO}

Intracellular NO detection in growing pollen tubes was accomplished by the incubation in $10 \mu \mathrm{M} 4,5$ diaminofluorescein diacetate (DAF-2DA, Calbiochem) during 5 minutes [28]. Parallel samples were treated with $200 \mu \mathrm{M}$ c-PTIO for 30 minutes prior to the treatment with DAF-2DA as above.

Observations were carried out in a Nikon C1 confocal microscope using an Ar-488 laser source and small pinhole $(30 \mu \mathrm{m})$. The fluorescent signal was recorded in green color with the EZ-C1 Nikon (Gold version 2.10 build 240) software.

\section{Determination of pollen viability.}

A few drops of propidium iodide (PI, Sigma-Aldrich), at a concentration of $10 \mu \mathrm{g} / \mathrm{ml}$ in water was added to the pollen sample just before imaging. Observations were carried out in a Nikon $\mathrm{C} 1$ confocal microscope using a He-Ne $(543 \mathrm{~nm})$ laser source and the signal was recorded in red color.

\section{Fluorimetric determination of NO released from pollen during in vitro germination.}

Released NO was determined as previously described [30; 31]. Briefly, aliquots (2ml) of samples at different times of germination $(1,2,4,6$ and $8 \mathrm{~h}$ ) were incubated with $10 \mu \mathrm{M} \mathrm{DAF}-2$ for 15 min and the germination medium was collected by centrifugation and filtration. Triazolofluorescein (DAF-2T)derived fluorescence of the supernatants was determined in a spectrofluorimeter PTI (Microbeam) with 
excitation and emission wavelengths of 493 and $515 \mathrm{~nm}$ (10 nm band width), respectively. Parallel samples were also exposed to $200 \mu \mathrm{M}$ c-PTIO since the beginning of the in vitro germination procedure. Three independent experiments were carried out.

\section{GSNO quantification by liquid chromatography-electrospray mass spectrometry (LC-ES/MS)}

GSNO detection was achieved as described previoulsy [32] with minor modifications. Olive mature or germinated pollen samples $(50 \mathrm{mg}$ each) were vigorously vortexed during $1 \mathrm{~min}$ in the presence of $2 \mathrm{ml}$ of $0.1 \mathrm{M} \mathrm{HCl}$. Homogenates were centrifuged at $15,000 \mathrm{~g}$ for $20 \mathrm{~min}$ at $4{ }^{\circ} \mathrm{C}$. The supernatants were collected and filtered through $0.22 \mu \mathrm{m}$ polyvinylidene fluoride filters and immediately analyzed. All procedures were carried out at $4^{\circ} \mathrm{C}$ and in the dark to avoid potential degradation of the analyte. The LCES/MS system consisted of a Waters Allience 2695 HPLC system connected to a Micromass Quattro micro API triple quadrupole mass spectrometer (Waters Corporation). HPLC was carried out using an Atlantis T3 $3 \mu \mathrm{m} 2.1 \times 100 \mathrm{~mm}$ column obtained from the Waters Corporation. The Micromass Quattro Micro API mass spectrometer was used in positive electrospray ionization mode. Mass spectrometry parameters were optimized by continuous infusion of GSNO in $0.1 \mathrm{M} \mathrm{HCl}$ [32]. Three independent experiments were performed.

\section{Preparation of protein extracts}

For protein extraction, mature pollen as well as germinated pollen from cultivar 'Picual' were powdered in liquid nitrogen and re-suspended in extraction buffer [1 M Tris- $\mathrm{HCl}$ (pH 7.4), $2 \mathrm{M}$ urea, $60 \mathrm{mM}$ DTT, 1\% Triton X-100 and protease inhibitor cocktail (Sigma-Aldrich)] to a proportion of $15 \mathrm{ml}$ solution per gram of fresh tissue. After centrifugation at $13,000 \mathrm{~g}$ for $20 \mathrm{~min}$ at $4{ }^{\circ} \mathrm{C}$, the supernatants were filtered through a $0.22 \mu \mathrm{m}$ mesh and used for Western blot analysis. For detection of $S$-nitrosylated proteins, the extraction buffer was replaced [50 mM HEPES buffer (pH 7.2), 0.25 M sucrose, $1 \mathrm{mM}$ EDTA]

\section{Western blotting analyses of protein $S$-nitrosylation and Tyr-nitration.}

The samples were separated on precast $4-20 \%$ acrylamide gels (Bio-Rad). Protein separation was conducted for $45 \mathrm{~min}$ at $120 \mathrm{~V}$. Gels were then subjected to transference to polyvinylidene difluoride (PVDF) membrane before Western blot analysis. Parallel sets of gels were stained with colloidal Coomassie blue.

The membranes were incubated for $1 \mathrm{~h}$ at room temperature in $5 \%(\mathrm{w} / \mathrm{v})$ defatted dry milk-TBST (blocking buffer). After blocking was completed, membranes were rinsed with copious amounts of TBST and exposed to rabbit anti-3-NT primary antibody (Millipore) solution (1:1,000 dilution in blocking buffer) and rabbit anti-S-nitrosoCys primary antibody (Sigma-Aldrich) solution (1:1,500 dilution in blocking buffer), respectively and incubated overnight at $4{ }^{\circ} \mathrm{C}$. After incubation, the membranes were 
washed with TBST and incubated with an anti-rabbit IgG Fc-peroxide conjugated secondary antibody (Sigma-Aldrich) at 1:5,000 dilution in TBST for $1 \mathrm{~h}$ at room temperature. Reactive bands were visualized using an ECL Plus detection kit (Bio-Rad) according to the manufacturer's instructions, and images were captured on a ChemiDoc Imager system (Bio-Rad).

\section{Immunoprecipitation with the anti-3-NT- and anti-S-nitrosoCys- antibodies}

Proteins from 'Picual' mature pollen homogenates, as well as germinated pollen extracts of the same cultivar (25 mg of protein) were suspended into lysis buffer that contained $50 \mathrm{mM}$ Tris- $\mathrm{HCl}(\mathrm{pH} 7.4), 150$ $\mathrm{mM} \mathrm{NaCl}, 1 \mathrm{mM}$ EDTA, and $0.1 \%(\mathrm{v} / \mathrm{v})$, Nonidet P-40. The suspension was then briefly sonicated and centrifuged at 13,000 g for $10 \mathrm{~min}$. The supernatant was collected, adjusted to a concentration of $25 \mathrm{mg} / \mathrm{ml}$ protein, and subjected to immunoprecipitation with either the anti-3-NT polyclonal (Millipore) or the anti-S-nitrosoCys polyclonal (Sigma-Aldrich) antibodies mentioned above, which were previously conjugated to protein A agarose beads (Sigma-Aldrich) for $2 \mathrm{~h}$ at room temperature with constant slow shaking. The beads were then collected by centrifugation and exhaustively washed (five times) with the lysis buffer. Finally, samples were re-suspended into Laemmli sample buffer and boiled for 5 min to dissociate the antigen-antibody complex. The solution (100 $\mu$ l) was then loaded onto the 4-20\% SDSPAGE gels (Bio-Rad) and subjected to electrophoretic separation. The gels were visualized by colloidal Coomassie staining, and bands of interest were excised and subjected to nano LC-MS analysis. Finally, replicas of these last gels were subjected again to Western blotting using the same antibodies as a final check.

Nano LC - MS analysis of bands.

LC was performed using an NanoAcquity nano-HPLC (Waters), equipped with a Waters BEH C18 nanocolumn (200mm x 75 um ID, 1.8um), A chromatographic ramp of $15 \mathrm{~min}$ (5 to $60 \%$ acetonitrile -ACN-) was used with a flow rate of $300 \mathrm{nl} / \mathrm{min}$. A $15 \mathrm{~min} 85 \% \mathrm{ACN}$ wash step was applied after linear gradient. Mobile phase A was water containing $0.1 \% \mathrm{v} / \mathrm{v}$ formic acid, while mobile phase B was ACN containing $0.1 \% \mathrm{v} / \mathrm{v}$ formic acid. A lock mass compound [Glu1]-Fibrinopeptide B (100fmol/ul) was delivered by an auxiliary pump of the LC system at $500 \mathrm{nl} / \mathrm{min}$ to the reference sprayer of the NanoLockSpray (Waters) source of the mass spectrometer. $0.5 \mathrm{ug}$ of each sample were loaded for each run.

Mass spectrometry: A Synapt G2Si ESI Q-Mobility-TOF spectrometer (Waters) equipped with an ion mobility chamber (T-Wave-IMS) was used for high definition data acquisition analyses. All analyses were performed in positive mode ESI. Data were post-acquisition lock mass corrected using the double charged monoisotopic ion of [Glu1]-Fibrinopeptide B. Accurate mass LC-MS data were collected in HDDA mode that enhances signal intensities using the ion mobility separation step.

Database search:

Database searching was performed using MASCOT 2.2.07 (Matrixscience, London, UK) against an ad 
hoc in house made database filled only with entries corresponding to Olea europaea L. For protein identification the following parameters were adopted: carbamidomethylation of cysteines (C) as fixed modification and oxidation of methionines (M) as variable modifications, $15 \mathrm{ppm}$ of peptide mass tolerance, 0.2 Da fragment mass tolerance and up to 3 missed cleavage points, Peptide charges of +2 and +3 .

\section{Measurements tools}

Pollen tubes length was measured using ImageJ software (http://rsb.info.nih.gov/ij/). Both average and standard deviation were calculated after measurement of a minimum of 100 tubes corresponding to three independent experiments. To analyze the germination rate, 300 grains/experiment along three independent experiments were considered.

The intensity of the green fluorescence was quantified by using the Nikon EZ-C1 viewer (3.30) software. Both average and standard deviation were calculated after measurement of a minimum of ten images corresponding to three independent experiments.

\section{$\underline{\text { Statistical analysis }}$}

The Kolmogorov-Smirnov test was used to test the normality of data. Statistical significance of fluorimetric quantification of NO release into the culture medium and quantification of GSNO in olive pollen through the in vitro germination was determined by ANOVA followed by a Bonferroni multiple comparison test. Differences in the pollen tube length among samples were assessed by Kruskal-Wallis one-way ANOVA analysis followed by the Dunn multiple-comparison Z-value test. One-way ANOVA analysis, followed by the Duncan multiple range test, was used to assess differences in the germination rate among samples. All analyses were performed using SPSS v.23 software (IBM, USA).

\section{Pollen and pistil transcriptomes}

Transcriptomes used in this work were generated from pollen and pistil Sanger sequences and Roche/454 reads at different developmental stages, as described in ReprOlive (http://reprolive.eez.csic.es) [33]. Pollen transcriptome comprises 27,823 transcripts of which 21,607 (77.7\%) had an orthologue according to the annotation software Full-LengtherNext (Seoane et al., submitted). A minimum of 11,672 were different transcripts and 1,976 of them contain a complete ORF.

\section{Predictive $S$-nitrosylation and Tyr-nitration tools}


A bibliographic search of freely available bioinformatics tools for $S$-nitrosylation and Tyr-nitration prediction was performed. Several of these tools were evaluated and tested on the basis of the chance of coping with a large number of sequences and their effectiveness in predicting prone to nitrosylation sites in comparison to other tools. Two of these tools were finally selected: iSNO-AAPair and GPS-YNO2. The tool iSNO-AAPair [34] predicts cysteine $S$-nitrosylation sites in proteins. It has a user-friendly accessible web-server for prediction (http://app.aporc.org/iSNO-AAPair) and supports large input files. On the other hand, GPS-YNO2 [35] predicts tyrosine nitration sites in proteins. Although this tool is freely available at http://yno2.biocuckoo.org for using in a web browser, authors recommend downloading the local version for inputs greater than 1,000 sequences. The pollen transcriptome was independently subjected to analysis with each tool. ISNO-AAPair was used on the web site, whereas GPS-YNO2 software was downloaded, installed and executed on a local machine. In both cases the input consists of a file with protein sequences in FASTA format. Default parameters (low-restrictive) were used.

ISNO-AAPair output is an HTML file, whereas GPS-YNO2 output is a plain text file. These output files were parsed to generate easier to handle tab-delimited text files, including the protein identifier in ReprOlive and the position/s prone to modification. Annotation data coming from ReprOlive was also added, including the closest plant orthologue, the ORF status (full-length or incomplete coded proteins) and a definition, all provided by Full-LengtherNext, as well as the identifier of the corresponding orthologue in Arabidopsis thaliana from TAIR10 database for further functional analyses.

Authors recommend using as input the entire sequence of the query protein for better predictions rather than fragments. However, since the number of complete proteins in both transcriptomes was relatively low, the entire collection of coded proteins according to Full-LengtherNext (including incomplete proteins) was used in the analyses (21,607 sequences from pollen).

As additional trials, predictions were repeated now using supplementary tools available (GPS-SNO: http://sno.biocuckoo.org, and SNOSite: http://csb.cse.yzu.edu.tw/SNOSite/, both for S-nitrosylation prediction) and again with GPS-YNO2 for Tyr-nitration. Now, the highest restrictive threshold settings in all predictive tools were used, in order to compare the outputs of the different tools and to assess the robustness of the predictions.

\section{Functional annotation and enrichment analysis}

The enrichment analysis tools AgriGO [36] and GeneCodis [37; 38; 39] were used to go into a comprehensive understanding of biological meaning, e.g. determining the most significant biological processes, molecular functions and cellular components in which the predicted modifiable proteins take part. Lists of TAIR identifiers from Supplementary Tables 1 and 4 (see Tables 1 and 4 in Ref [40]) were used as input file. In the case of AgriGO (http://bioinfo.cau.edu.cn/agriGO/analysis.php) singular enrichment analyses (SEA) were carried out using the following settings: the Arabidopsis genome TAIR10 as reference, hypergeometric as statistical test, Hochberg (FDR) as multi-test adjustment method, 0.01 as the significance level, 10 as minimum number of mapping entries and Plant GO slim as gene 
ontology type. Regarding GeneCodis (http://genecodis.cnb.csic.es/analysis), singular enrichment analyses were also performed using the following combination of parameters: default GeneCodis3 list of genes as reference, the lowest level of GO annotations, 10 as minimum number of genes, hypergeometric test as method to compute p-values and FDR as method to correct p-values. The same kind of enrichment analysis was carried out with both tools, including quite similar combinations of parameters and statistical treatment. However, the graphical representation of the final results varies, and depending on the purpose, a certain type of graph can be more appropriate than other. Furthermore, GeneCodis supports enrichment analyses of KEGG pathways.

\section{RESULTS}

Cell localization of NO production during in vitro pollen germination and simultaneous assessment of pollen viability

NO was produced during olive pollen in vitro germination (Figure 1, A-D). The fluorescence was significantly reduced in the presence of the NO-scavenger c-PTIO (Figure 1, A'-D'). The majority of the hydrated grains presented green fluorescence, mainly labeling the apertural regions of the pollen grain (Figure 1A and Figure 2A-A'”). NO-signal was non-homogeneously distributed throughout the cytoplasm of the vegetative cell. Curiously, some of the pollen grains lacked of any labeling. Simultaneous double staining of the hydrated pollen grains with the DAF-2DA fluorochrome and propidium iodide revealed that most pollen grains stained by PI (non-viable grains) were not stained by DAF-2DA, and the other way round (Figure 2A-A'”).

Approximately $3 \mathrm{~h}$ after the initiation of the in vitro culture, we observed the maximum number of pollen grains displaying pollen tubes starting its emergence, and these short pollen tubes also displayed intense fluorescence due to the presence of NO (Figure 1B). The fluorescence was accumulated in the cytoplasm of the pollen tube, with several spots of high intensity present in the cytoplasm. After $5 \mathrm{~h}$ of in vitro germination, green fluorescence was normally restricted to the pollen tube, with a spotty pattern (Figure 2B, B' '). Most pollen grains showing NO-derived fluorescence did not display simultaneous labeling of the nuclei by PI (Figure 2). At longer culture times, fluorescence was extended to the whole pollen tube (Figure 1D).

NO distribution along the growing pollen tube (Figure 1B' $-\mathrm{C}$ ') ) was not uniform. Thus, fluorescence was progressively accumulated in the subapical zone (Figure 1B', C', ) although longer culture time exhibited fluorescence extended to the whole tube (Figure 1D' '). The use of NO scavenger c-PTIO caused not only a drastic reduction in overall fluorescence, but also a more homogeneous distribution of fluorescence, where the maximum located at the subapical zone almost disappeared (Figure 1C').

\section{Determination of NO release into the culture medium during in vitro germination}


Fluorimetric determination of extracellular NO at different times of in vitro germination showed that olive pollen was able to release NO to the culture medium, as observed by a logarithmic increase in the fluorescence intensity from the beginning of the hydration process (Figure 3). This NO release was maximum after $3 / 4 \mathrm{~h}$ of in vitro germination, and then rapidly decayed. The addition of the NO scavenger c-PTIO caused a reduction in the DAF-2 derived fluorescence in the culture medium, which remained at basal levels through the whole culture.

\section{GSNO quantification}

GSNO, a mobile reservoir of NO, was detected in olive pollen grain through the in vitro germination as well as in the germination medium (Figure 4). Pollen GSNO content displayed an increasing trend throughout the culture, which was well correlated with the NO content previously reported. Furthermore, GSNO released to the medium was not detected up to $5 \mathrm{~h}$ of in vitro germination, reaching maximal intensity later, after $14 \mathrm{~h}$ of culture (Figure 4 ).

\section{Effects of NO donors and scavengers during in vitro germination}

When olive pollen was challenged for NO donors and scavenger, exogenous NO was shown to decrease pollen germination and tube elongation rates. Results (Figure 5) showed that both pollen germination (as percentage over the control) and tube length (in micrometers) showed an exponential decrease after the treatment with either SNP or GSNO. This reduction was dependent on the concentration of the NO donor used, suggesting the presence of a dose-response relationship. GSNO was able to provoke a shaper effect in both pollen germination and pollen tube length than SNP, even at lower concentrations.

In the absence of donors, c-PTIO itself was able to slightly increase both the rate of germination and the pollen tube length, although the difference was not significant. This chemical also reduced the inhibitory effects on pollen germination of both NO donors assayed, although its counteracting effect was especially evident when GSNO was used. In the same way, the greater effect on tube length caused by GSNO was cushioned by c-PTIO, whereas the counteracting effect after SNP was not so evident.

Pollen tubes treated with NO donors were not only shorter but also displayed altered morphology such as balloon tips, isodiametric expansions of the tube, broader tubes or double onset of germination (Figure 6). Furthermore, the NO scavenger c-PTIO was not only unable to totally revert the effects detected in morphology but also, swollen tips were detected in samples treated with c-PTIO only. In the absence or donors, c-PTIO was found to provoke a small percentage (14.5\%) of slightly balloon-shaped tips (Figure $6)$. 
A total of 21,607 predicted proteins derived from the olive pollen transcriptome were tested throughout iSNO-AAPair using default settings. From those, 9,266 (42.9\%) of the proteins were identified as prone to cysteine $S$-nitrosylation. Since many of these proteins share the same orthologue, the number of proteins when unique orthologues were considered was reduced to 5,899 (27.3\%). Supplementary Table 1 (see Table 1 in Ref [40]) shows predicted cysteine $S$-nitrosylation sites in proteins from pollen transcriptome obtained with iSNO-AAPair. In addition to the Protein ID (protein identifier in ReprOlive database), abundant complementary information provided includes the position/s of amino acid/s prone to nitrosylation, susceptibility of the protein to simultaneous tyrosine nitration, open reading frame (ORF) status (full-length or incomplete proteins) and definition, respectively, all provided by Full-LengtherNext annotation tool. Finally, corresponding Arabidopsis thaliana orthologues on TAIR10 database are also reported.

For comparison purposes, similar predictions were carried out by using the alternative tools GPS-SNO and SNOSite for Cys-nitrosylation prediction. In both cases, the highest restrictive thresholds available were used. The number of predicted proteins prone to this PTM raised to 15,285 for SNOSite, and lowered to 7,788 for GPS-SNO (supplementary Tables 2 and 3) (see Tables 2 and 3 in Ref [40]).

Singular enrichment analysis of GO biological processes in which putatively $S$-nitrosylated proteins are involved include a wide panel of categories, with a larger proportion of proteins involved in several metabolic processes, response to several stresses (Cd, salt), transport, etc. Interestingly, numerous proteins tagged as involved in pollen development, pollen tube growth and pollen germination were identified (Figure 7). As regard to molecular functions, numerous categories were defined, including protein, ATP, Zn, nucleic acid and nucleotide binding, as well as diverse enzyme activities. Putative proteins subjected to this PTM were predicted to have many distinct cell localizations (plasma membrane, nucleus, chloroplast, membrane, plasmodesmata, cytoplasm...), whereas most relevant KEEG pathways involved include biosynthesis of secondary metabolites, with the larger proportion of genes involved, followed by other relevant pathways like protein processing in endoplasmic reticulum, oxidative phosphorylation, and the metabolism of starch, sucrose, amino acids, nucleotides, pyruvate, and glycerophospholipids among others (Figure 7).

In silico prediction, functional annotation and enrichment analysis of Tyr-nitration sites in olive pollen proteins

A total of 21,607 predicted proteins derived from the olive pollen transcriptome were tested throughout GPS-YNO2. From those, 12,977 (60.1\%) of the proteins were identified as prone to Tyrosine nitration. Since many of these proteins share the same orthologue, the number of proteins when unique orthologues were considered was reduced to 7,883 (36.5\%). Supplementary Table 4 (see Table 4 in Ref [40]) shows predicted Tyr-nitration sites in proteins from pollen transcriptome obtained with GPS-YNO2. As per $S$ nitrosylation, in addition to the Protein ID (protein identifier in ReprOlive database), abundant complementary information provided includes the position $/ \mathrm{s}$ of amino acid/s prone to nitration, 
susceptibility of the protein to simultaneous $S$-nitrosylation, open reading frame (ORF) status (full-length or incomplete proteins) and definition, respectively, all provided by Full-LengtherNext annotation tool. Finally, corresponding Arabidopsis thaliana orthologues on TAIR10 database are also provided.

Also for comparison purposes, similar prediction was carried out by using the GPS-YNO2 tool; however the highest restrictive threshold available was now used. In this case, the number of predicted proteins prone to this PTM lowered to 10,836 (supplementary Table 5) (see Tables 5 in Ref [40]).

As described above for predicted $S$-nitrosylated proteins, singular enrichment analysis of GO biological processes in which putatively Tyr-nitrated proteins are involved include a wide panel of categories, with a larger proportion of proteins involved in protein phosphorylation, several metabolic processes, response to several stresses (Cd, salt), transport, etc. Also, coincidentally with $S$-nitrosylated proteins, a relevant number of proteins tagged as involved in pollen development, and pollen tube growth were identified (Figure 8). Regarding molecular functions, numerous categories were defined, including protein, ATP, $\mathrm{Zn}$, nucleic acid (DNA and RNA), nucleotide binding, as well as diverse enzyme activities. Putative proteins subjected to this PTM were predicted to have many distinct cell localizations, with a large proportion of proteins predicted as components of the plasma membrane, nucleus, chloroplast, membrane, plasmodesmata...). Finally, most relevant KEEG pathways involved include biosynthesis of secondary metabolites with the larger proportion of genes involved, followed by other relevant pathways like protein processing in endoplasmic reticulum, spliceosome, and the metabolism of starch, sucrose, amino acids, nucleotides, etc. among others (Figure 8).

A comparison carried out between the proteins prone to cysteine $S$-nitrosylation and those susceptible to tyrosine nitration revealed that a significant percentage of proteins (almost 40\%) could be subject to both types of modification (Figure 9).

\section{Immunodetection and immunoprecipitation of $S$-nitrosylated and Tyr-nitrated proteins from olive pollen extracts}

Mature pollen extracts, as well as proteins extracts of pollen grains subjected to in vitro pollen germination were assessed for the presence of S-nitrosylated proteins by Western blotting. Numerous bands reacting to an antibody to $S$-nitrosylated Cys residues were identified in the extracts. A clearly distinctive pattern was observed in the extract corresponding to the germinated pollen (Figure $10 \mathrm{~A}, \mathrm{~B}$ ). Immunoprecipitation with the antibody, and further Western blotting analysis carried out also with the same antibody allowed the isolation of multiple bands, with a good correlation in sizes with those positive bands identified in the whole pollen extracts (Figure 10C). Most prominent bands after immunoprecipitation were extracted and subjected to nano-LC-MS identification.

\section{Immunoprecipitation of Tyr-nitrated proteins}


A similar approach to the one described above was used to assess for the presence of Tyr-nitrated proteins by Western blotting. Now, bands harboring nitrated Tyr residues were identified with the help of a specific antibody to this chemically-modified amino acid. Again, differential patterns of reacting bands were observed in the extracts of both mature olive pollen, and the germinated pollen (Figure $11 \mathrm{~A}, \mathrm{~B}$ ). Figure 11C shows the results of immunoprecipitation and further Western blotting analysis carried out with this last antibody. Similarly, most prominent bands after immunoprecipitation were extracted and subjected to nano-LC-MS identification.

\section{Nano-LC-MS identification of $S$-nitrosylated/Tyr-nitrated proteins}

MS analysis of bands reacting to the SNO-Cys and the anti-3-NT antibodies after immunoprecipitation yielded a high number of identifications, which are listed in Supplementary Tables 6 and 7 (see Tables 6 and 7 in Ref [40]). These included 491 and 345 matches, respectively, including members of numerous protein families. Searches of these proteins throughout the iSNO-AAPair, GPS-SNO, SNOSite and GPSYNO2 databases, respectively identified large percentages of these proteins as putatively $S$-nitrosylated or Tyr-nitrated proteins, even though the highest thresholds were used for such predictions. Venn representations of the intersecting matches between the different predictions and the validated proteomic data are represented in Figures 12 and 13.

\section{DISCUSSION}

The importance of NO in sexual plant reproduction has been demonstrated and discussed during the last years, although still many questions remain to be answered. NO, as well as ROS, are present in the pollen grain and the stigmatic papillae of diverse species where they play important signaling roles [21; 24]. In this work we have demonstrated that NO production and distribution in olive pollen exhibit characteristic models, and are directly connected to physiological pollen performance. Thus, patterning of NO presence and distribution is clearly different between viable and non-viable pollen grains, and between hydrated pollen grains and the growing pollen tubes. In pollen tubes, an intense reorganization of the cytoplasm occurs, triggered by hydration, calcium influx and polar growth. Scientific community is nowadays beginning to build interesting models trying to integrate the information available on NO signal in the pollen tube $[18 ; 19 ; 27 ; 28 ; 41]$. It must be highlighted that there are clear evidences about the interaction between NO accumulation and $\mathrm{Ca}^{2+}$ influx $[18 ; 19 ; 20 ; 25]$. Furthermore, a direct mechanism for NO regulation of the pollen cytoskeleton via actin $S$-nitrosylation has been recently suggested [42].

During olive pollen tube growth progression, the distribution of NO reaches a maximum in the subapical domain, whereas the fluorescence was progressively excluded from the grain. In lily pollen, fluorescence is seen in the cytosol associated to round cytoplasmic organelles (peroxisomes), with less intensity in the apical domain [28]. Contrary, in pollen tubes from $P$. bungeana, the fluorescence was distributed throughout nearly the entire tube, except in the tip region. After treatment with c-PTIO, NO-dependent signal was distributed throughout nearly the entire tube and authors suggest that this due to the clear zone disruption [19]. In a similar way, the treatments of olive pollen with NO scavengers caused both a decrease in fluorescence as well as a more homogenously distribution of the signal. In cucumber, ROS 
and NO were specifically localized at the pollen tube tip, whereas at later growing times the fluorescence was found all along the tube's length and even inside the pollen grain [10].

Within a regulatory and functional context, we also found that NO donors (SNP and GSNO) inhibited olive pollen germination and tube growth as it occurs in the species of angiosperms previously studied. Thus, treatment of cucumber pollen with GSNO and SNP inhibited both pollen germination and pollen tube growth in a dose-dependent manner, although pollen germination rate seems to be increased in the presence of the NO-scavenger c-PTIO [10]. Nevertheless, c-PTIO had no effect on either olive pollen germination or tube growth in the absence of donors but can significantly reduce the inhibitory effects of both SNP and GSNO, as it has previously reported for Paulownia tomentosa pollen [43]. Furthermore, the addition of c-PTIO in olive pollen caused the emergence of balloon tips in a small fraction of pollen tubes as it had been previously observed in lily [19; 28]. Although germination and pollen tube growth rates were partially rescued by using the NO scavenger, abnormal tube phenotypes caused by the addition of NO donors in olive pollen were still found in these samples in a lesser degree. A previous study connecting NO and the organization of actin filaments in pollen tubes of $P$. bungeana showed similar morphological alterations by using NO donors/scavengers [19]. As suggested by other studies, GSNO could be more efficient than SNP in reducing germination and pollen tube length due to a higher capability of NO production. Using two different concentrations of donors ( $0.5 \mathrm{mM}$ and $1 \mathrm{mM})$, around 3-times higher concentration of NOx was detected for GSNO than for SNP [44]. A later work [44] reached a similar conclusion using $0.2 \mathrm{mM}$ of either donors. On the other hand, it should be noticed that maybe not only the amount but also the redox form of $\mathrm{NO}$ generated varies among donors $\left(\mathrm{NO}^{+}\right.$for $\mathrm{SNP}^{-}$ and $\mathrm{NO}^{\circ}$ for GSNO). Such differences could be sensed by plant cells in different ways. This could explain at least in part, the different effects reported for SNP and GSNO in different works [44; 46].

These results suggested than a fine adjustment of NO production should be accomplished throughout physiological development of the pollen tube, and that such fine tune could not be fully emulated by the rough approximation of using chemical donors/scavengers. In a recent study focused in cold-mediated pollen tube growth inhibition [20], authors showed that a NO donor was able to mimic the effect of the stress and caused disruption of the cytoplasmic $\mathrm{Ca}^{2+}$ gradient, increase in ROS content and acidification of cytoplasmic $\mathrm{pH}$. At the cytological level, abnormalities were also observed.

Simultaneous double staining of the hydrated pollen grains with the DAF-2DA fluorochrome and PI allowed some additional conclusions to be drawn, as the second is commonly used for identifying dead cells in a population [47]. A direct relationship between viable pollen grains and NO production seems to occur in olive pollen. However, there was no direct evidence of the opposite situation, as non-viable pollen grains might or not produce NO. The reasons for such correlation between viable pollen grains and NO production are unknown, and have not been pointed out before in the literature according to our knowledge, as most studies focused in viable pollen grains only [10]. Pollen viability has been described to be affected by related parameters like ozone pollution and increased UV-B radiation [48; 49]. Such factors are known to increase the production of ROS like $\mathrm{O}_{2}{ }^{--}$and $\mathrm{H}_{2} \mathrm{O}_{2}$, leading to increased lipid peroxidation and decreased activities of antioxidants. Production of NO could become affected in a similar way. Alternatively, the absence of fluorescent signal in those grains affected by low viability 
might be the result of at least one of different factors including lack of esterase activity able to hydrolize DAF-2DA, inability to produce NO, or NO diffusion through the plasma membrane of the vegetative cell due to the lack of integrity in this structure combined to the high diffusion capacity of this molecule. PI has been proposed to accumulate in non-viable cells only, due to the absence of fully comprehensive membrane. As the result, nuclei in these cells appear intensely labeled, oppositely to viable (with an intact plasma membrane) cells [50]. Classical assays of pollen viability based in the combined use of fluorescein diacetate (FDA) and PI [51] could not be carried out because of the coincidence in the excitation/emission wavelengths of FDA and DAF-2DA.

The release of NO molecules from germinated pollen grains to the culture medium showed in this work is also novel and arises important questions. The release upon hydration suggests a key role for NO during pollen-pistil interactions, where this molecule may act as a signal regulating the interaction pollen-pistil, as it has been previously indicated [21;23;52]. In the same way, NO released by pollen tubes could serve as a signaling molecule in a necessary cross-talk between the pollen tube and the transmitting tissue of the pistil [22]. At this developmental stage, a continuous signals interchange between both partners (pollen and pistil) it is needed, since cross-communication has to be carried out in a tight time frame [53].

The technique used for GSNO detection (LC-ES/MS) has been shown to provide a consistent quantification [32]. Surprisingly, GSNO levels in germinated olive pollen reached almost ten-fold the reported GSNO content in pepper leaves by these authors. The function of plant endogenous GSNO in the response to biotic and abiotic stress has being highlighted but the study of its abundance, regulation or distribution during plant development is still scarce [14]. This work shows that the increase in the inner pollen NO content along the first $4 \mathrm{~h}$ of in vitro pollen germination highly correlates with the presence of GSNO in the pollen grains along this time. However, after this period, the sharp decrease in the inner pollen NO content described here becomes coincidental with a slight increase of GSNO within the pollen grains, but most significantly with a sharp increase in the GSNO detected in the culture medium. Such changes are compatible with a balanced generation of GSNO from the existing NO during the early period (up to $4 \mathrm{~h}$ ), which is rapidly increased after $4 \mathrm{~h}$. The high amount of GSNO produced after this time contributes both to the sudden presence of GSNO in the culture medium and to the abrupt diminution of the inner pollen NO concentration. This premise should be further investigated.

NO short half-life makes difficult its direct measurement. Several methods have been developed in the last years looking for reliable detection and quantification, being DAF-2 and the cell permeable version DAF-2DA the most frequent probes. However, their reliability has been questioned [30; 31]. In this study, GSNO was selected as the NO donor in addition to SNP, considering the results set out in different studies. A work focused in the effect of different widely used NO-donors showed that SNP induces PCD and suppression of ROS scavenging system being thereof less efficient in NO production than GSNO [43]. The commonly used donor SNP has been described to produced cyanide [54], which could act as an inhibitor of diverse enzymes and provoke effects on biological systems, acting together with NO effects. Opposite, a different study suggested that SNP is a more efficient NO generator than GSNO [55]. A different study delved into the stability of NO donors and authors tried to highlight that the process of donor decomposition depends on numerous external factors, which should be considered [45]. Here, both 
SNP and GSNO were used as NO donors with similar effects on the occurrence of pollen tube alterations. However, GSNO was able to provoke a shaper effect when compared to SNP.

Transcriptomic strategies can be envisaged to particularly analyze the involvement of NO in a particular biological process. As an example, differential gene expression analyses have been used to explore the involvement of NO in C. sinensis pollen tube growth at low temperature [20]. As per olive pollen, the ReprOlive database (http://reprolive.eez.csic.es/olivodb/) [33] is an easy-to-use olive tree (Olea europaea L.) database containing its reproductive transcriptome obtained from pollen and stigma (both together and separately). We have used the information stored and organized within this olive database to design new in silico strategies to explore the potential of both $S$-nitrosylation and Tyr-nitration in the olive pollen.

Recent research in multiple plant systems has been focused to perform comparative analysis of the plant $S$-nitrosoproteome in both unstressed plants and in plants exposed to different stress stimuli [56; 57]. $S$ nitrosylation in pollen seems to represent a relevant modification, affecting key gene products which include numerous allergens (many of them also displaying enzymatic activities), NADPH oxidase [58], actin [59], and different molecules putatively involved in self-incompatibility [60], among others. Also, recent work has updated the involvement of the NO-PTM protein tyrosine nitration in the development and stress response in plants [61]. Preliminary identification of proteins prone to these PTMs is considered essential for further identification of those proteins subjected to protein modifications either because of greater RNS content, or because they represent targets for cell-signaling events. Massive transcriptome assessment as performed here in pollen for the first time represents in our view a valuable tool, highly complementary to the proteomic approaches and the point use of in silico prediction methods currently in use [13]. Prior massive computational prediction of candidate proteins for $S$-nitrosylation was carried out previously [62] using a whole Arabidopsis thaliana transcriptome. Relevant similarities were detected between such study and the present one (i.e. the large number of candidate proteins for $S$ nitrosylation as well as the detection of many common protein targets). Functional and comparative analysis of such set of proteins may help to understand the importance of RNS in plant and particularly in pollen physiology. As an example, our GO enrichment analysis reveals the large variety of potential targets of $S$-nitrosylation and Tyr-nitration and the wide variety of molecular functions, cell localizations, and biochemical pathways potentially involved. Of course, experimental determination and validation of the $S$-nitrosoproteome and nitroproteome under different physiological scenarios is necessary. As an example, we describe here relevant differences in the $S$-nitrosylated and Tyr-nitrated protein profiles of mature pollen and germinated pollen, and we have experimentally identified a huge number of specific proteins subjected to these modifications, member of numerous protein families, and with multiple functions. Interestingly, actin peptides have been thoroughly identified among the proteins recognized by the anti-S-nitrosoCys- antibodies. Actin has been demonstrated to be modified by $S$-nitrosylation, and this PTM results in interferences with actin polymerization, able to cause severe disturbances in actin cytoskeleton distribution and function [59]. The integrity of the F-actin cytoskeleton has been described as a key factor controlling self-compatibility in Nicotiana alata [63; 64].

The use of predictive algorithms to foresee the proteins subjected to PTMs is always a matter of controversy, as the use of low thresholds may lead to false positives, whereas the use of highly restrictive 
thresholds may conduct to lose relevant information. The present work has combined different algorithms for prediction, and different thresholds in order to minimize, or at least to assess the extension of these variables in a certain degree. Moreover, the extensive determination of protein identities by using highlysensitive, experimental methods like immunoprecipitation and nano-LC-MS and further back-crossing of the information as shown here may help to us to reach a supported prediction. Thus, a large proportion of the S-nitrosylated and Tyr-nitrated identified proteins by MS (31 and 67\%, respectively) were also predicted simultaneously by all the corresponding algorithms used here, and much higher percentages of these proteins were predicted at least by one of the corresponding tools used (86 and 79\%, respectively). On the other hand, 14 and $21 \%$ of the S-nitrosylated and Tyr-nitrated proteins identified by MS were not predicted by any of the algorithms used here. This can be the result of low sensitivity of the programs used, which are constantly improved on the basis of the published literature, or simply to protein contaminations or non-specific interactions of the antibodies used during the immunoprecipitation procedures, combined with the extreme sensitivity of nano-LC-MS methods. These facts indicate that much work has yet to be carried out to precisely and experimentally determine whether the predicted gene products or even the identified proteins are really modified by the proposed PTMs in vivo.

Another interesting point to be discussed is that the NO emission by olive pollen grains may have a particular effect on the epithelium cells of the respiratory airways in humans. This molecule has been shown to play a potential role triggering human allergy symptoms [23; 52]. S-protein nitrosylation has been shown to alter allergenicity profiles of several allergens [65] increasing their allergenic capacity. Also, enhanced allergenic response has been described after nitration of pollen aeroallergens by nitrate ion in conditions simulating the liquid water phase of atmospheric particles [66]. Therefore, olive pollen (one of the most important allergen sources in Mediterranean countries), and displaying a complex pattern of potentially NO-PTM proteins, is likewise to also suffer alterations in its allergenicity as a consequence of these protein modifications. As an example, Supplementary tables 6 and 7 include numerous olive pollen allergens (i.e. Ole e 5, Ole e 8, Ole e 10 and Ole e 11) as well as heterologous allergens (i.e. Che a 1, Pru ar 1 and Fra e 1) (see Tables 6 and 7 in Ref [40]).

An important unanswered question as regard to pollen biology is the identification of the potential sources of NO in this plant material. Two NO enzymatic sources have been suggested to act in pollen. Using inhibitors of NOS-like activity, the NO production was reduced in cypress, P. tomentosa or camellia pollen [27; 43; 67]. On the other hand, pollen from the nial ${ }^{-}$nia2 $2^{-}$double mutant (with reduced NR activity) showed no differences on NO-related fluorescence when compared to the wild type, although the situation was different when ATP analogue were capable of stimulate NO production just in the wild type [41]. At present, no similar experiments have been performed in olive pollen. Hence, there is the need of a deeper study of NO origin in pollen from different species.

\section{CONCLUSIONS}


NO production by in vitro germinating olive pollen was imaged in situ with the fluorescent probe DAF-2 DA, which allowed to determine a positive correlation between NO production and pollen viability, likely associated to plasma membrane integrity. The growing pollen tubes release NO to the culture medium over the early steps of pollen germination, which turns into GSNO once the pollen tubes emerge massively from pollen grains during the culture. Pollen germination and pollen tube growth are both diminished by the addition of NO donors, and these effects are partially reverted by the specific NOscavenger c-PTIO. The bioinformatics assessment of the potential length of NO-PTMs ( $S$-nitrosylation and Tyr-nitration) in olive pollen demonstrated that a large percentage of gene products are prone to these PTMs, taking part of many different biological processes, molecular functions, cell distributions and metabolic pathways. Several of these proteins have been monitored and identified after using experimental methods including the use of specific antibodies and proteomic approaches.

\section{ACKNOWLEDGEMENTS}

This work was supported by ERDF co-funded projects BFU2011-22779, BFU2016-77243-P, RTC-20154181-2 and RTC-2016-4824-2 (MINECO), P2011-CVI-7487 (Junta de Andalucía) and 201540E065 (CSIC).

\section{LEGENDS TO THE FIGURES}

Figure 1. Localization of intracellular NO generation during in vitro pollen germination by using DAF2DA. Upper panel: the localization of NO is shown by green fluorescence (A-D). Samples preincubated with c-PTIO (NO scavenger) showed a noticeable reduction in the green fluorescence (middle panel, A'D'). Bottom panel: Quantification (Nikon EZ-C1 v. 3.90) of the fluorescence intensity in arbitrary units (A.U.) of a representative pollen tube, from aperture $(0 \mu \mathrm{m})$ along tube length (B' '-D' '). PG: pollen grain; PT: pollen tube. Images represent DAF-2DA fluorescence merged to DIC bright field view of the samples. Bars $=10 \mu \mathrm{m}$

Figure 2. NO production versus pollen-viability in hydrated pollen grains (A-A') and germinated/nongerminated pollen grains (B-B'). NO production was imaged by DAF-2DA staining (green fluorescence), whereas pollen viability was assessed by propidium iodide (red fluorescence staining of pollen nuclei). Ex: exine; PG: pollen grain; PT: pollen tube; GN: generative nucleus; VN: vegetative nucleus. * Putatively non-viable pollen. Bars $=10 \mu \mathrm{m}$

Figure 3. DAF-2 fluorimetric detection and quantification of NO release into the culture medium during in vitro pollen germination. Data are the means $\pm \mathrm{SD}(n=3$ in triplicate). Statistical significance was determined by ANOVA followed by a Bonferroni multiple comparison test. *Indicates that the mean is 
significantly different from that of sample at t:0h; \# indicates that the mean is significantly different from the equivalent sample incubated with c-PTIO at $P<0.05$

Figure 4. LC-ES/MS detection and quantification of $S$-nitrosoglutathione (GSNO) in olive pollen through the in vitro germination as well as in the germination medium by LC-ES/MS. MP: mature pollen; GP: germinated pollen; M: culture medium. Data are the means of three independent experiments. Statistical significance was determined by ANOVA followed by a Bonferroni multiple comparison test. *Indicates that the mean is significantly different from that of sample at $\mathrm{t}: 0 \mathrm{~h}$ at $P<0.05$.

Figure 5. Effect of SNP and GSNO (NO donors) on pollen germination and pollen tube length. Exogenous NO decreases pollen germination rates (A, B) and decreases pollen tube lengths (C, D) in a doses-dependent manner. Such effects were partially reverted by the simultaneous addition of the NO scavenger c-PTIO. Pollen germination percentages over the control and pollen tube length were determined after $5 \mathrm{~h}$ incubation of olive pollen in different concentration of SNP (A, C) or GSNO (B, D) with or without $200 \mu \mathrm{M}$ c-PTIO. Data are the means of three independent experiments $(\mathrm{n}=100$ in triplicate). Statistical significance for pollen tube length was determined by Kruskal-Wallis One-Way ANOVA analysis of variance and the Kruskal-Wallis Multiple-Comparison Z-Value Test. One-way ANOVA was used for the analysis of germination rates followed by with Duncan's Multiple Range Test. *Indicates that the mean is significantly different from the control (untreated sample) at $P<0.05$; \# indicates that the mean is significantly different from the equivalent sample incubated with c-PTIO at $P<$ 0.05

Figure 6. Distinctive features of pollen tubes germinated in vitro in the presence of NO-donors and scavengers. CP: callose plugs; PG: pollen grain; PT: pollen tube. Arrowheads: balloon-shaped apex; arrows: isodiametric expansions of the pollen tube; dots: broader pollen tubes; asterisk: double onset of germination. Bars $=50 \mu \mathrm{m}$.

Figure 7. Gene Ontology terms distribution of the putative cysteine $S$-nitrosylated proteins from pollen transcriptome obtained with iSNO-AAPair. The distribution of biological processes, molecular functions, cellular components and KEGG pathways was assessed. Number of genes is indicated for each category. The graphs were prepared using the Genecodis software.

Figure 8. Gene Ontology terms distribution of the putative Tyr-nitrated proteins from pollen transcriptome obtained with GPS-YNO2. The distribution of biological processes, molecular functions, 
cellular components and KEGG pathways was assessed. Number of genes is indicated for each category. The graphs were prepared using the Genecodis software.

Figure 9. Venn diagrams for comparisons between proteins prone to cysteine $S$-nitrosylation predicted by iSNO-AAPair and those prone to tyrosine nitration according to GPS-YNO2 in pollen (http://bioinfogp.cnb.csic.es/tools/venny).

Figure 10. Immunodetection of $S$-nitrosylated proteins from olive pollen extracts. Protein extracts of mature and germinated pollen (Coomassie staining in A) were assessed for the presence of $S$-nitrosylated proteins by Western blotting (B) and immunoprecipitation (C).

Figure 11. Immunodetection of proteins from olive pollen extracts. Protein extracts of mature pollen and germinated pollen (Coomassie staining in A) were assessed for the presence of Tyr-nitrated proteins by Western blotting (B) and immunoprecipitation (C).

Figure 12. Venn representations of the intersecting matches between the different predictions and the validated proteomic data: number of transcripts/gene products identified by ISNO-AAPAIR, GPS-SNO (high threshold), SNOSite (high threshold) and proteomic data, as prone to S-nitrosylation.

Figure 13. Venn representations of the intersecting matches between the different predictions and the validated proteomic data: number of transcripts/gene products identified by GPS-yno2 (medium -defaultthreshold), GPS-yno2 (high threshold) and proteomic data, as prone to Tyr-nitration.

\section{REFERENCES}

[1] S. Hayat, S.A. Hasan, M. Mori, Q. Fariduddin, A. Ahmad, Nitric oxide: chemistry, biosynthesis, and physiological role, Nitric Oxide in Plant Physiology, Wiley-VCH Verlag GmbH \& Co., KGaA, Weinheim 2009, pp. 1-15.

[2] K.J. Gupta, A.R. Fernie, W.M. Kaiser, J.T. van Dongen, On the origins of nitric oxide, Trends Plant Sci 16 (2011) 160-8.

[3] M. Yu, L. Lamattina, S.H. Spoel, G.J. Loake, Nitric oxide function in plant biology: a redox cue in deconvolution, New Phytol 202 (2014) 1142-56.

[4] L.A. Mur, J. Mandon, S. Persijn, S.M. Cristescu, I.E. Moshkov, G.V. Novikova, M.A. Hall, F.J. Harren, K.H. Hebelstrup, K.J. Gupta, Nitric oxide in plants: an assessment of the current state of knowledge, AoB Plants 5 (2013) pls052. 
[5] P. Domingos, A.M. Prado, A. Wong, C. Gehring, J.A. Feijo, Nitric oxide: a multitasked signaling gas in plants, Mol Plant 8 (2015) 506-20.

[6] J. Astier, C. Lindermayr, Nitric oxide-dependent posttranslational modification in plants: an update, Int J Mol Sci 13 (2012) 15193-208.

[7] L. Sanz, P. Albertos, I. Mateos, I. Sanchez-Vicente, T. Lechon, M. Fernandez-Marcos, O. Lorenzo, Nitric oxide (NO) and phytohormones crosstalk during early plant development, J Exp Bot 66 (2015) 2857-68.

[8] M. Delledonne, NO news is good news for plants, Curr Opin Plant Biol 8 (2005) 390-6.

[9] M. Delledonne, J. Zeier, A. Marocco, C. Lamb, Signal interactions between nitric oxide and reactive oxygen intermediates in the plant hypersensitive disease resistance response, Proc Natl Acad Sci U S A 98 (2001) 13454-9.

[10] J. Sirova, M. Sedlarova, J. Piterkova, L. Luhova, M. Petrivalsky, The role of nitric oxide in the germination of plant seeds and pollen, Plant Sci 181 (2011) 560-72.

[11] L. Freschi, Nitric oxide and phytohormone interactions: current status and perspectives, Front Plant Sci 4 (2013) 398.

[12] J.K. Abat, R. Deswal, Posttranslational modifications of proteins by nitric oxide: a new tool of metabolome regulation, Nitric Oxide in Plant Physiology, Wiley-VCH Verlag GmbH \& Co. KGaA 2009, pp. 189-201.

[13] I. Kovacs, C. Lindermayr, Nitric oxide-based protein modification: formation and site-specificity of protein S-nitrosylation, Front Plant Sci 4 (2013) 137.

[14] F.J. Corpas, J.D. Alche, J.B. Barroso, Current overview of S-nitrosoglutathione (GSNO) in higher plants, Front Plant Sci 4 (2013) 126.

[15] F.J. Corpas, J.M. Palma, M. Leterrier, L.A. del Río, J.B. Barroso, Nitric Oxide and abiotic stress in higher plants, Nitric Oxide in Plant Physiology, Wiley-VCH Verlag GmbH \& Co. KGaA 2009, pp. 51-63.

[16] M. Airaki, M. Leterrier, R.M. Mateos, R. Valderrama, M. Chaki, J.B. Barroso, L.A. Del Rio, J.M. Palma, F.J. Corpas, Metabolism of reactive oxygen species and reactive nitrogen species in pepper (Capsicum annuиm L.) plants under low temperature stress, Plant Cell Environ 35 (2012) 281-95.

[17] J.B. Barroso, R. Valderrama, F.J. Corpas, Immunolocalization of S-nitrosoglutathione, Snitrosoglutathione reductase and tyrosine nitration in pea leaf organelles, Acta Physiol Plant 35 (2013) 2635-2640.

[18] A.M. Prado, R. Colaco, N. Moreno, A.C. Silva, J.A. Feijo, Targeting of pollen tubes to ovules is dependent on nitric oxide (NO) signaling, Mol Plant 1 (2008) 703-14.

[19] Y. Wang, T. Chen, C. Zhang, H. Hao, P. Liu, M. Zheng, F. Baluska, J. Samaj, J. Lin, Nitric oxide modulates the influx of extracellular $\mathrm{Ca}^{2+}$ and actin filament organization during cell wall construction in Pinus bungeana pollen tubes, New Phytol 182 (2009) 851-62.

[20] W. Wang, X. Sheng, Z. Shu, D. Li, J. Pan, X. Ye, P. Chang, X. Li, Y. Wang, Combined cytological and transcriptomic analysis reveals a nitric oxide signaling pathway involved in cold-inhibited Camellia sinensis pollen tube growth, Front Plant Sci 7 (2016) 456.

[21] A. Zafra, M.I. Rodriguez-Garcia, J.D. Alche, Cellular localization of ROS and NO in olive reproductive tissues during flower development, BMC Plant Biol 10 (2010) 36.

[22] S.M. McInnis, R. Desikan, J.T. Hancock, S.J. Hiscock, Production of reactive oxygen species and reactive nitrogen species by angiosperm stigmas and pollen: potential signalling crosstalk?, New Phytol 172 (2006) 221-8.

[23] I.D. Wilson, S.J. Hiscock, P.E. James, J.T. Hancock, Nitric oxide and nitrite are likely mediators of pollen interactions, Plant Signal Behav 4 (2009) 416-8.

[24] A. Zafra, J.D. Rejon, S.J. Hiscock, J.D. Alche, Patterns of ROS accumulation in the stigmas of angiosperms and visions into their multi-functionality in plant reproduction, Front Plant Sci 7 (2016) 1112

[25] K.A. Wilkins, J. Bancroft, M. Bosch, J. Ings, N. Smirnoff, V.E. Franklin-Tong, Reactive oxygen species and nitric oxide mediate actin reorganization and programmed cell death in the selfincompatibility response of papaver, Plant Physiol 156 (2011) 404-16.

[26] I. Serrano, M.C. Romero-Puertas, M. Rodriguez-Serrano, L.M. Sandalio, A. Olmedilla, Peroxynitrite mediates programmed cell death both in papillar cells and in self-incompatible pollen in the olive (Olea europaea L.), J Exp Bot 63 (2012) 1479-93.

[27] Y.H. Wang, X.C. Li, Q. Zhu-Ge, X. Jiang, W.D. Wang, W.P. Fang, X. Chen, X.H. Li, Nitric oxide participates in cold-inhibited Camellia sinensis pollen germination and tube growth partly via cGMP in vitro, PLoS One 7 (2012) e52436. 
[28] A.M. Prado, D.M. Porterfield, J.A. Feijo, Nitric oxide is involved in growth regulation and reorientation of pollen tubes, Development 131 (2004) 2707-14.

[29] M. M'rani-Alaoui, A. Castro, J. Alché, W. Wang, M.C. Fernández, M.I. Rodríguez-García, Expression of Ole e 1, the major olive pollen allergen, during in-vitro pollen germination, Acta Hort 586 (2002) 465-468.[30] J.F. Leikert, T.R. Räthel, C. Müller, A.M. Vollmar, V.M. Dirsch, Reliable in vitro measurement of nitric oxide released from endothelial cells using low concentrations of the fluorescent probe 4,5-diaminofluorescein, FEBS Letters 506 (2001) 131134.

[31] E. Planchet, W.M. Kaiser, Nitric oxide (NO) detection by DAF fluorescence and chemiluminescence: a comparison using abiotic and biotic NO sources, J Exp Bot 57 (2006) 3043-3055.

[32] M. Airaki, L. Sanchez-Moreno, M. Leterrier, J.B. Barroso, J.M. Palma, F.J. Corpas, Detection and quantification of S-nitrosoglutathione (GSNO) in pepper (Capsicum annuum L.) plant organs by LC-ES/MS, Plant Cell Physiol 52 (2011) 2006-15.

[33] R. Carmona, A. Zafra, P. Seoane, A.J. Castro, D. Guerrero-Fernandez, T. Castillo-Castillo, A. Medina-Garcia, F.M. Canovas, J.F. Aldana-Montes, I. Navas-Delgado, J.D. Alche, M.G. Claros, ReprOlive: a database with linked data for the olive tree (Olea europaea L.) reproductive transcriptome, Front Plant Sci 6 (2015) 625.

[34] Y. Xu, X.-J. Shao, L.-Y. Wu, N.-Y. Deng, K.-C. Chou, iSNO-AAPair: incorporating amino acid pairwise coupling into PseAAC for predicting cysteine S-nitrosylation sites in proteins, PeerJ 1 (2013) e171.

[35] Z. Liu, J. Cao, Q. Ma, X. Gao, J. Ren, Y. Xue, GPS-YNO2: computational prediction of tyrosine nitration sites in proteins, Mol Biosyst 7 (2011) 1197-204.

[36] Z. Du, X. Zhou, Y. Ling, Z. Zhang, Z. Su, agriGO: a GO analysis toolkit for the agricultural community, Nucleic Acids Res 38 (2010) W64-70.

[37] P. Carmona-Saez, M. Chagoyen, F. Tirado, J.M. Carazo, A. Pascual-Montano, GENECODIS: a webbased tool for finding significant concurrent annotations in gene lists, Genome Biol 8 (2007) R3.

[38] R. Nogales-Cadenas, P. Carmona-Saez, M. Vazquez, C. Vicente, X. Yang, F. Tirado, J.M. Carazo, A. Pascual-Montano, GeneCodis: interpreting gene lists through enrichment analysis and integration of diverse biological information, Nucleic Acids Res 37 (2009) W317-22.

[39] D. Tabas-Madrid, R. Nogales-Cadenas, A. Pascual-Montano, GeneCodis3: a non-redundant and modular enrichment analysis tool for functional genomics, Nucleic Acids Research 40 (2012) W478-W483.

[40] M.J. Jimenez-Quesada, R. Carmona, E. Lima-Cabello, J.A. Traverso, A.J. Castro, M.G. Claros, J.D. Alché, S-nitroso- and nitro- proteomes in the olive (Olea europaea L.) pollen. Predictive versus experimental data by nano-LC-MS. Data in Brief (submitted).

[41] S.A. Reichler, J. Torres, A.L. Rivera, V.A. Cintolesi, G. Clark, S.J. Roux, Intersection of two signalling pathways: extracellular nucleotides regulate pollen germination and pollen tube growth via nitric oxide, J Exp Bot 60 (2009) 2129-38.

[42] F. Zhao, A. Elkelish, J. Durner, C. Lindermayr, J.B. Winkler, R.F. Rusmall io, H. Behrendt, C. Traidl-Hoffmann, A. Holzinger, W. Kofler, P. Braun, C. von Toerne, S.M. Hauck, D. Ernst, U. Frank, Common ragweed (Ambrosia artemisiifolia L.): allergenicity and molecular characterization of pollen after plant exposure to elevated $\mathrm{NO}_{2}$, Plant Cell Environ 39 (2016) 147-64.

[43] J.M. He, X.L. Bai, R.B. Wang, B. Cao, X.P. She, The involvement of nitric oxide in ultraviolet-Binhibited pollen germination and tube growth of Paulownia tomentosa in vitro, Physiol Plant 131 (2007) 273-82.

[44] I. Murgia, M.C. de Pinto, M. Delledonne, C. Soave, L. De Gara, Comparative effects of various nitric oxide donors on ferritin regulation, programmed cell death, and cell redox state in plant cells, J Plant Physiol 161 (2004) 777-83.

[45] J. Floryszak-Wieczorek, G. Milczarek, M. Arasimowicz, A. Ciszewski, Do nitric oxide donors mimic endogenous NO-related response in plants?, Planta 224 (2006) 1363-72.

[46] J. Kovacik, P. Babula, B. Klejdus, J. Hedbavny, M. Jarosova, Unexpected behavior of some nitric oxide modulators under cadmium excess in plant tissue, PLoS One 9 (2014) e91685.

[47] K.H. Jones, J.A. Senft, An improved method to determine cell viability by simultaneous staining with fluorescein diacetate-propidium iodide, J Histochem Cytochem 33 (1985) 77-9.

[48] S. Wang, B. Xie, L. Yin, L. Duan, Z. Li, A.E. Eneji, W. Tsuji, A. Tsunekawa, Increased UV-B radiation affects the viability, reactive oxygen species accumulation and antioxidant enzyme activities in maize (Zea mays L.) pollen, Photochem Photobiol 86 (2010) 110-6. 
[49] S. Pasqualini, E. Tedeschini, G. Frenguelli, N. Wopfner, F. Ferreira, G. D'Amato, L. Ederli, Ozone affects pollen viability and $\mathrm{NAD}(\mathrm{P}) \mathrm{H}$ oxidase release from Ambrosia artemisiifolia pollen, Environ Pollut 159 (2011) 2823-30.

[50] C.-N. Huang, M. Cornejo, D. Bush, R. Jones, Estimating viability of plant protoplasts using double and single staining, Protoplasma 135 (1986) 80-87.

[51] Y. Lee, E.-S. Kim, Y. Choi, I. Hwang, C.J. Staiger, Y.-Y. Chung, Y. Lee, The Arabidopsis phosphatidylinositol 3-kinase is important for pollen development, Plant physiol 147 (2008) 1886-1897.

[52] J. Bright, S.J. Hiscock, P.E. James, J.T. Hancock, Pollen generates nitric oxide and nitrite: a possible link to pollen-induced allergic responses, Plant Physiol Biochem 47 (2009) 49-55.

[53] T. Dresselhaus, N. Franklin-Tong, Male-female crosstalk during pollen germination, tube growth and guidance, and double fertilization, Mole Plant 6 (2013) 1018-1036.

[54] P.C. Bethke, I.G. Libourel, V. Reinohl, R.L. Jones, Sodium nitroprusside, cyanide, nitrite, and nitrate break Arabidopsis seed dormancy in a nitric oxide-dependent manner, Planta 223 (2006) 805-12.

[55] L. Ederli, L. Reale, L. Madeo, F. Ferranti, C. Gehring, M. Fornaciari, B. Romano, S. Pasqualini, NO release by nitric oxide donors in vitro and in planta, Plant Physiol Biochem 47 (2009) 42-8.

[56] J. Begara-Morales, B. Sánchez-Calvo, M. Chaki, R. Valderrama, C. Mata-Pérez, F. Corpas, J. Barroso, Protein S-nitrosylation and S-glutathionylation as regulators of redox homeostasis during abiotic stress response, Redox state as a central regulator of plant-cell stress responses, Springer 2016, pp. 365-386.

[57] T. Tichá, L. Luhová, M. Petřivalský, Functions and metabolism of s-nitrosothiols and s-nitrosylation of proteins in plants: the role of GSNOR, Gasotransmitters in plants, Springer 2016, pp. 175200.

[58] H.T. Xie, Z.Y. Wan, S. Li, Y. Zhang, Spatiotemporal production of reactive oxygen species by NADPH oxidase is critical for tapetal programmed cell death and pollen development in Arabidopsis, Plant Cell 26 (2014) 2007-2023.

[59] M. Rodriguez-Serrano, D.M. Pazmino, I. Sparkes, A. Rochetti, C. Hawes, M.C. Romero-Puertas, L.M. Sandalio, 2,4-Dichlorophenoxyacetic acid promotes S-nitrosylation and oxidation of actin affecting cytoskeleton and peroxisomal dynamics, J Exp Bot 65 (2014) 4783-93.

[60] T. Haque, Investigating the targets and mechanisms regulating self incompatibility in Papaver rhoeas pollen, ( $\mathrm{PhD}$ thesis), University of Birmingham (2015).

[61] C. Mata-Perez, J.C. Begara-Morales, M. Chaki, B. Sanchez-Calvo, R. Valderrama, M.N. Padilla, F.J. Corpas, J.B. Barroso, Protein tyrosine nitration during development and abiotic stress response in plants, Front Plant Sci 7 (2016) 1699.

[62] M. Chaki, I. Kovacs, M. Spannagl, C. Lindermayr, Computational prediction of candidate proteins for S-nitrosylation in Arabidopsis thaliana, PLoS One 9 (2014) e110232.

[63] J.A. Roldan, H.J. Rojas, A. Goldraij, In vitro inhibition of incompatible pollen tubes in Nicotiana alata involves the uncoupling of the F-actin cytoskeleton and the endomembrane trafficking system, Protoplasma 252 (2015) 63-75.

[64] J.A. Roldan, H.J. Rojas, A. Goldraij, Disorganization of F-actin cytoskeleton precedes vacuolar disruption in pollen tubes during the in vivo self-incompatibility response in Nicotiana alata, Ann Bot 110 (2012) 787-95.

[65] F. Zhao, A. Elkelish, J. Durner, C. Lindermayr, J.B. Winkler, F. Ruëff, H. Behrendt, C. TraidlHoffmann, A. Holzinger, W. Kofler, P. Braun, C. von Toerne, S.M. Hauck, D. Ernst, U. Frank, Common ragweed (Ambrosia artemisiifolia L.): allergenicity and molecular characterization of pollen after plant exposure to elevated $\mathrm{NO}_{2}$, Plant, Cell \& Environment 39 (2016) 147-164.

[66] A. Ghiani, M. Bruschi, S. Citterio, E. Bolzacchini, L. Ferrero, G. Sangiorgi, R. Asero, M.G. Perrone, Nitration of pollen aeroallergens by nitrate ion in conditions simulating the liquid water phase of atmospheric particles, Sci Total Environ 573 (2016) 1589-1597.

[67] S. Pasqualini, M. Cresti, C. Del Casino, C. Faleri, G. Frenguelli, E. Tedeschini, L. Ederli, Roles for NO and ROS signalling in pollen germination and pollen-tube elongation in Cupressus arizonica, Biol Plant (2015) 1-10.

\section{SUPPLEMENTARY MATERIAL}

Supplementary Table 1 (see Table 1 in Ref [40]). Predicted cysteine S-nitrosylation sites in proteins from pollen transcriptome obtained with iSNO-AAPair. Protein id: protein identifier in ReprOlive 
database; S-nitrosylation position/s: position/s of amino acid/s prone to nitrosylation according to the tools: iSNO-AAPair, GPS-sno (high threshold) and SNOSite (high specificity level); Tyrosine nitration position/s: position/s of amino acid/s prone to tyrosine nitration according to the tool GPS-yno2 (medium and high thresholds); FLN ortholog, FLN status and FLN definition: the closest plant orthologue, ORF status (full-length or incomplete proteins) and definition, respectively, all provided by Full-LengtherNext; Tair ortholog: corresponding Arabidopsis thaliana orthologue on TAIR10 database.

Supplementary Table 2 (see Table 2 in Ref [40]). Predicted cysteine S-nitrosylation sites in proteins from pollen transcriptome obtained with GPS-sno (high threshold). Protein id: protein identifier in ReprOlive database; S-nitrosylation position/s: position/s of amino acid/s prone to nitrosylation according to the tools: iSNO-AAPair, GPS-sno (high threshold) and SNOSite (high specificity level); Tyrosine nitration position/s: position/s of amino acid/s prone to tyrosine nitration according to the tool GPS-yno2 (medium and high thresholds); FLN ortholog, FLN status and FLN definition: the closest plant orthologue, ORF status (full-length or incomplete proteins) and definition, respectively, all provided by Full-LengtherNext; Tair ortholog: corresponding Arabidopsis thaliana orthologue on TAIR10 database.

Supplementary Table 3 (see Table 3 in Ref [40]). Predicted cysteine S-nitrosylation sites in proteins from pollen transcriptome obtained with SNOSite (high specificity level). Protein id: protein identifier in ReprOlive database; S-nitrosylation position/s: position/s of amino acid/s prone to nitrosylation according to the tools: iSNO-AAPair, GPS-sno (high threshold) and SNOSite (high specificity level); Tyrosine nitration position/s: position/s of amino acid/s prone to tyrosine nitration according to the tool GPS-yno2 (medium and high thresholds); FLN ortholog, FLN status and FLN definition: the closest plant orthologue, ORF status (full-length or incomplete proteins) and definition, respectively, all provided by Full-LengtherNext; Tair ortholog: corresponding Arabidopsis thaliana orthologue on TAIR10 database.

Supplementary Table 4 (see Table 4 in Ref [40]). Predicted tyrosine nitration sites in proteins from pollen transcriptome obtained with GPS-YNO2 (medium -default- threshold). Protein id: protein identifier in ReprOlive database; Tyrosine nitration position/s: position/s of amino acid/s prone to tyrosine nitration according to the tool GPS-yno2 (medium and high thresholds); S-nitrosylation position/s: position/s of amino acid/s prone to nitrosylation according to the tools: iSNO-AAPair, GPSsno (high threshold) and SNOSite (high specificity level); FLN ortholog, FLN status and FLN definition: the closest plant orthologue, ORF status (full-length or incomplete proteins) and definition, respectively, all provided by Full-LengtherNext; Tair ortholog: corresponding Arabidopsis thaliana orthologue on TAIR10 database. 
Supplementary Table 5 (see Table 5 in Ref [40]). Predicted tyrosine nitration sites in proteins from pollen transcriptome obtained with GPS-YNO2 (high threshold). Protein id: protein identifier in ReprOlive database; Tyrosine nitration position/s: position/s of amino acid/s prone to tyrosine nitration according to the tool GPS-yno2 (medium and high thresholds); S-nitrosylation position/s: position/s of amino acid/s prone to nitrosylation according to the tools: iSNO-AAPair, GPS-sno (high threshold) and SNOSite (high specificity level); FLN ortholog, FLN status and FLN definition: the closest plant orthologue, ORF status (full-length or incomplete proteins) and definition, respectively, all provided by Full-LengtherNext; Tair ortholog: corresponding Arabidopsis thaliana orthologue on TAIR10 database.

Supplementary Table 6 (see Table 6 in Ref [40]). MS identification of bands reacting to the SNO-Cys antibody after immunoprecipitation, and searches of these proteins throughout the tools for prediction of S-nitrosylation [iSNO-AAPair, GPS-sno (high threshold) and SNOSite (high level of specificity)] and Tyr-nitration [GPS-YNO2, medium and high level].

Supplementary Table 7 (see Table 7 in Ref [40]). MS identification of bands reacting to the anti-3-NT antibody after immunoprecipitation, and searches of these proteins throughout the tools for prediction of S-nitrosylation [iSNO-AAPair, GPS-sno (high threshold) and SNOSite (high level of specificity)] and Tyr-nitration [GPS-YNO2, medium and high level].

DECLARATION OF INTEREST. The authors declare no financial, personal or other conflict of interest 


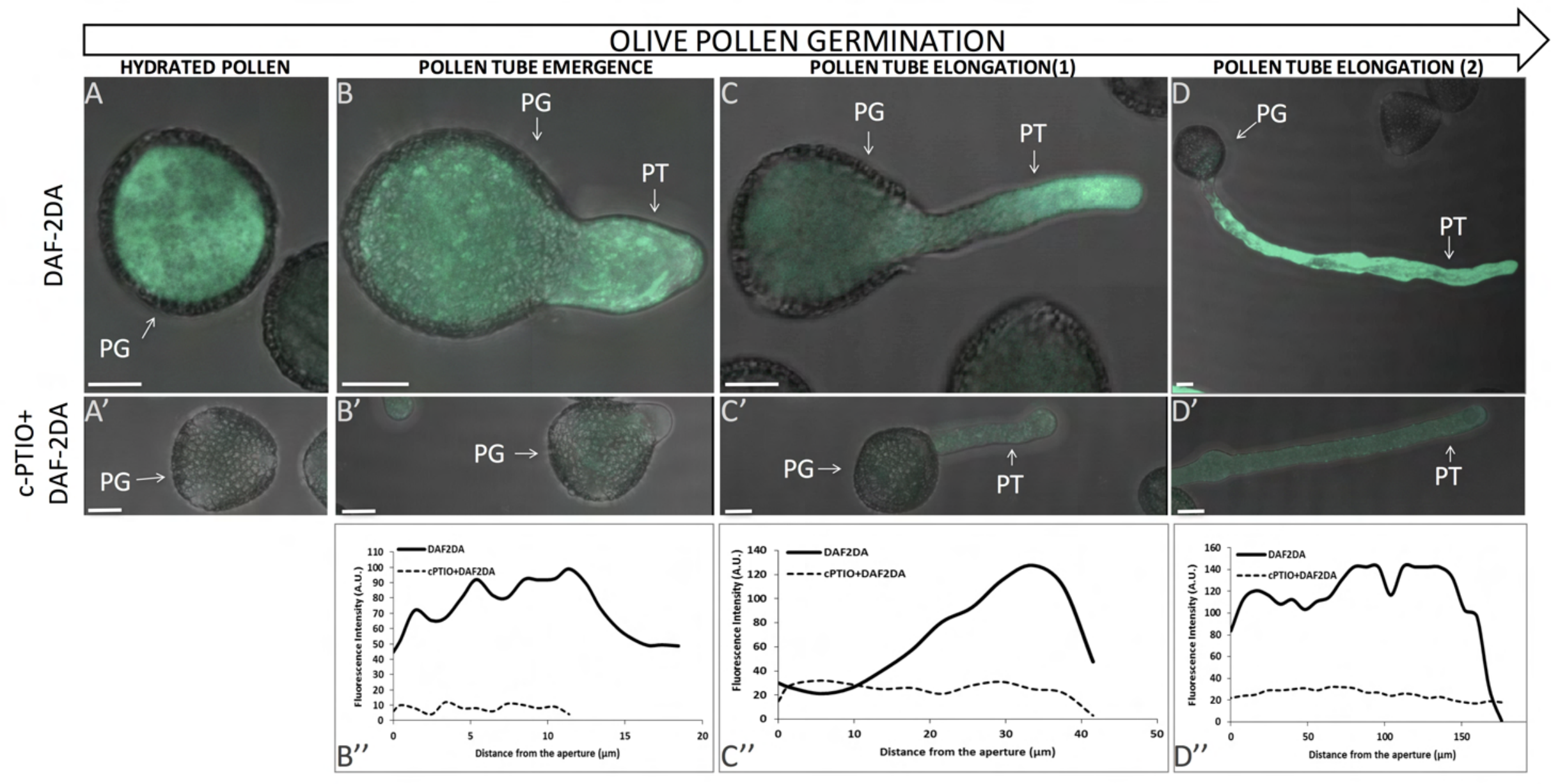




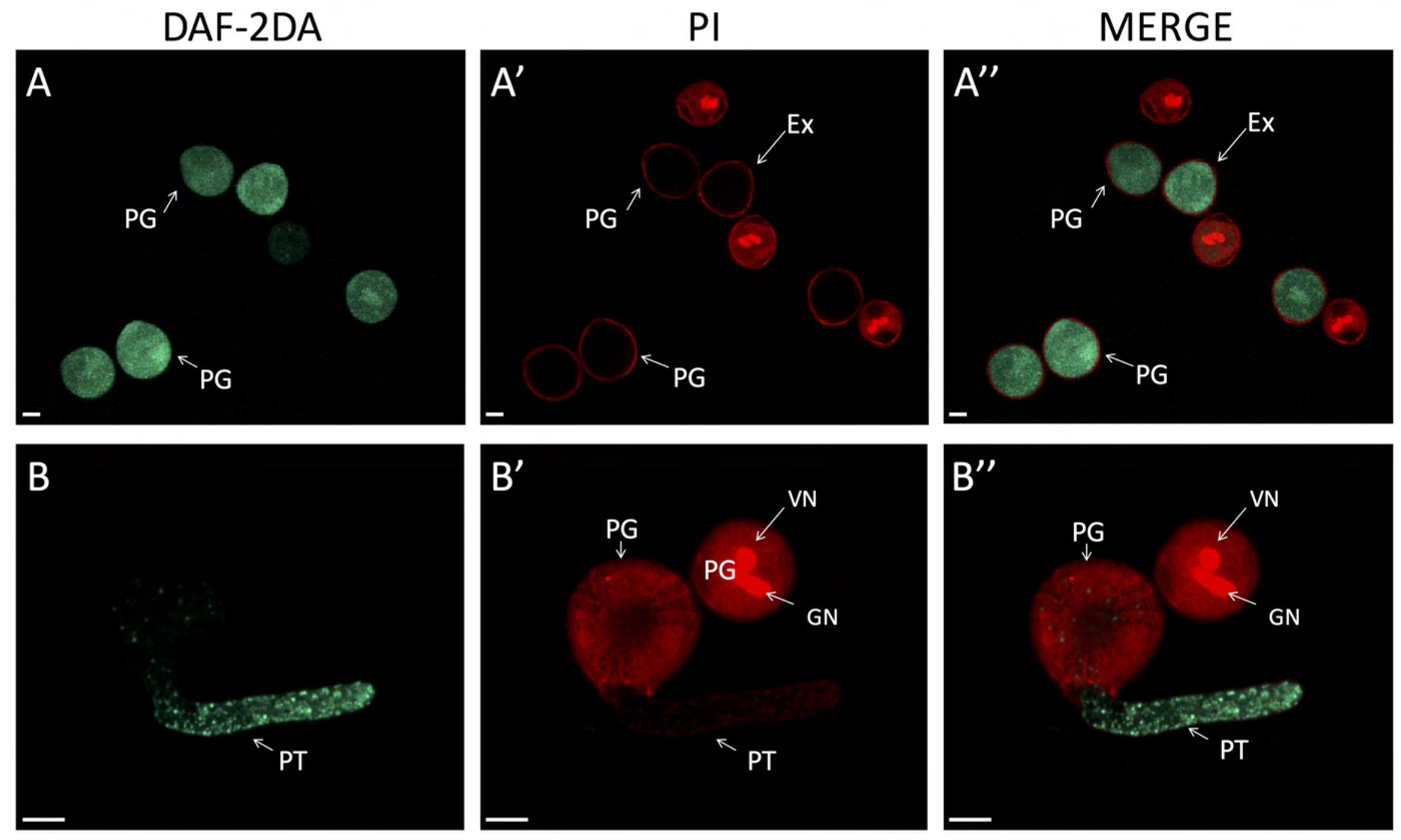




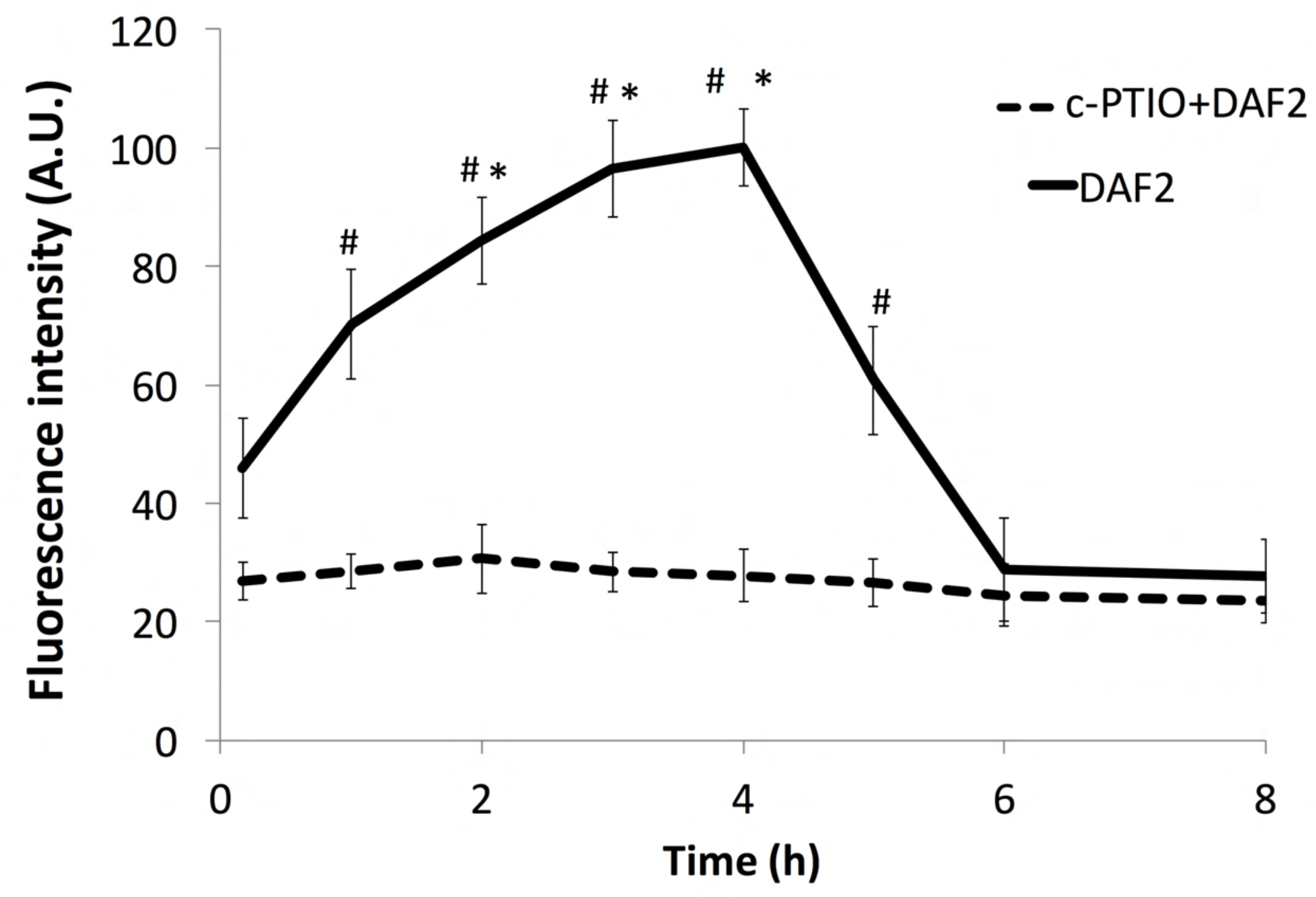




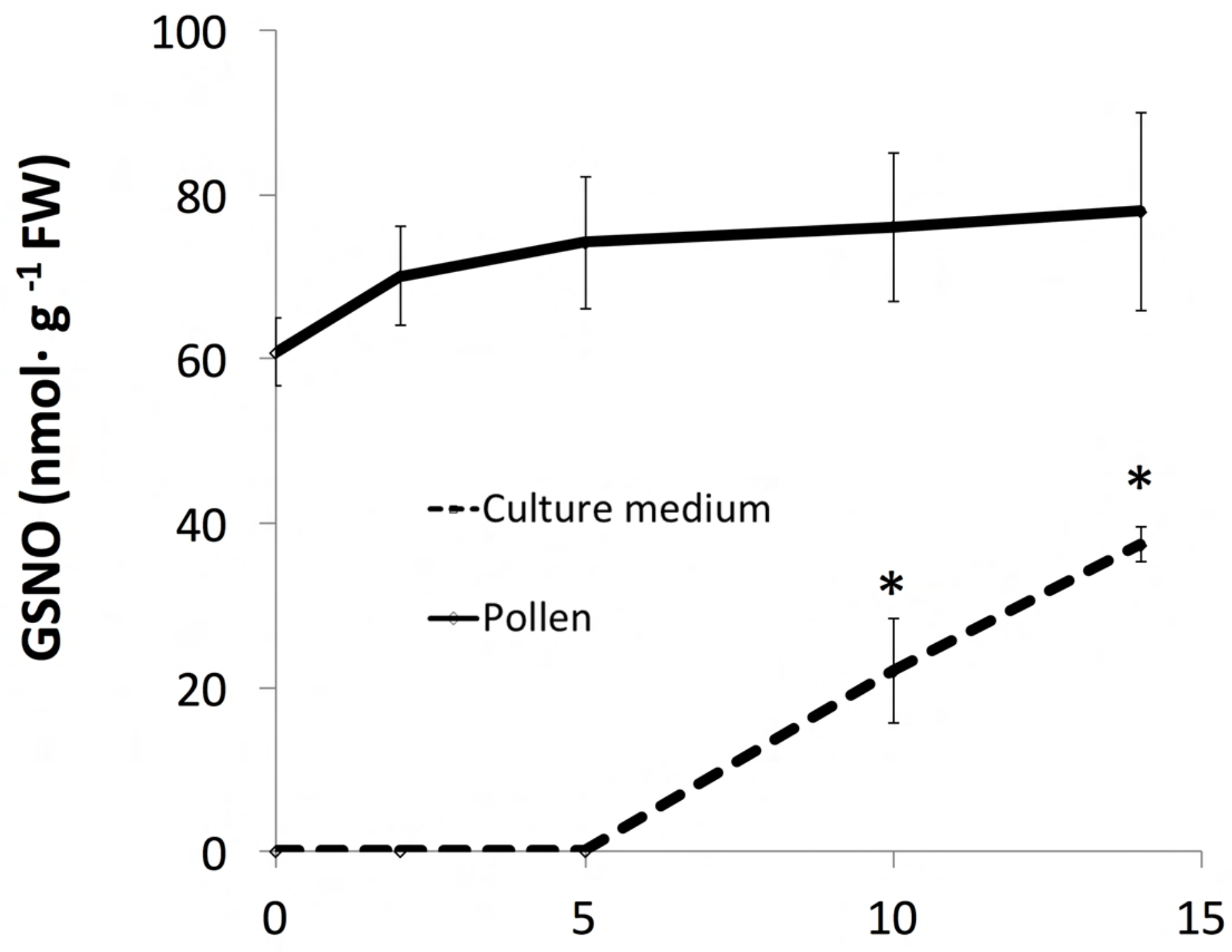



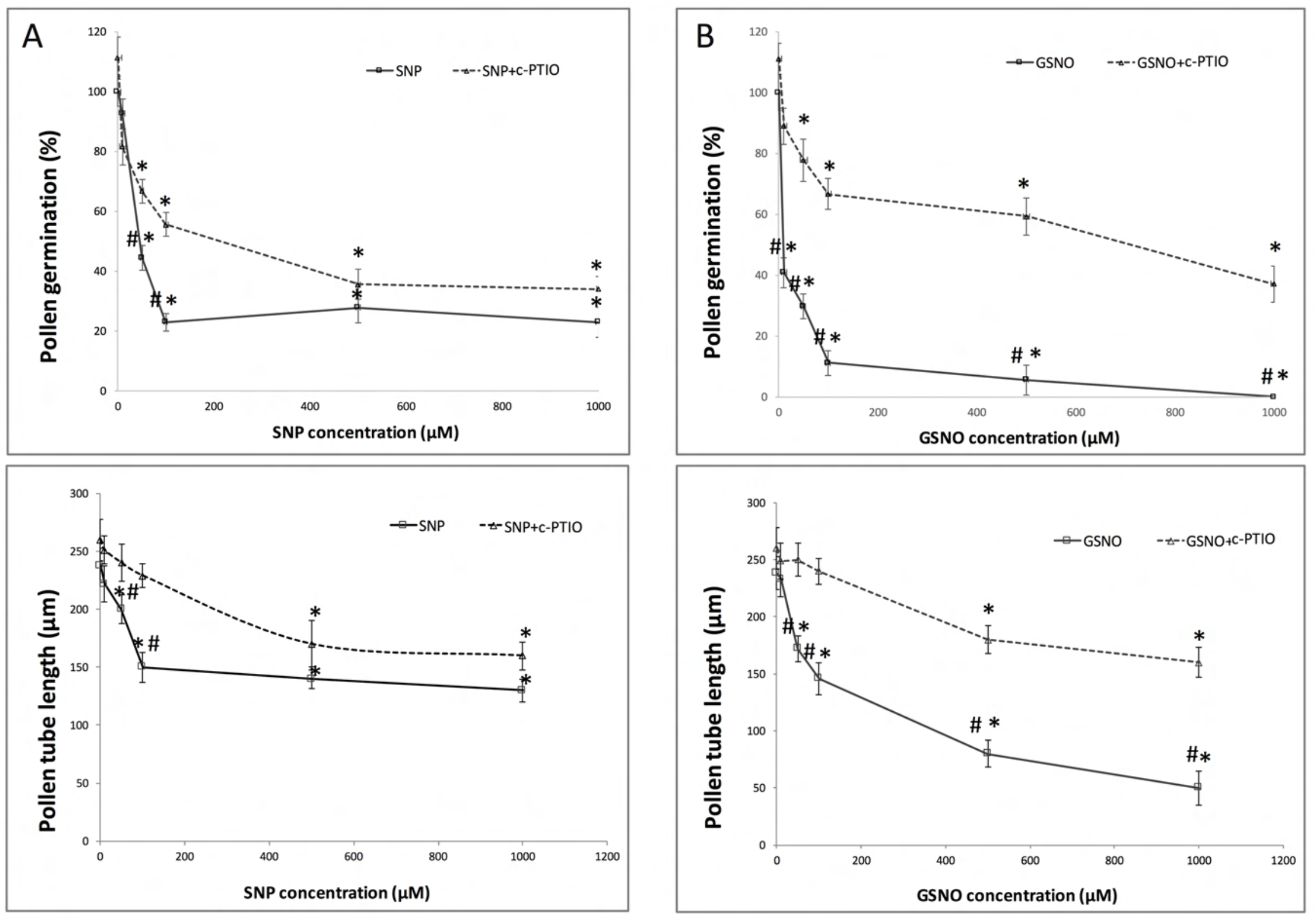


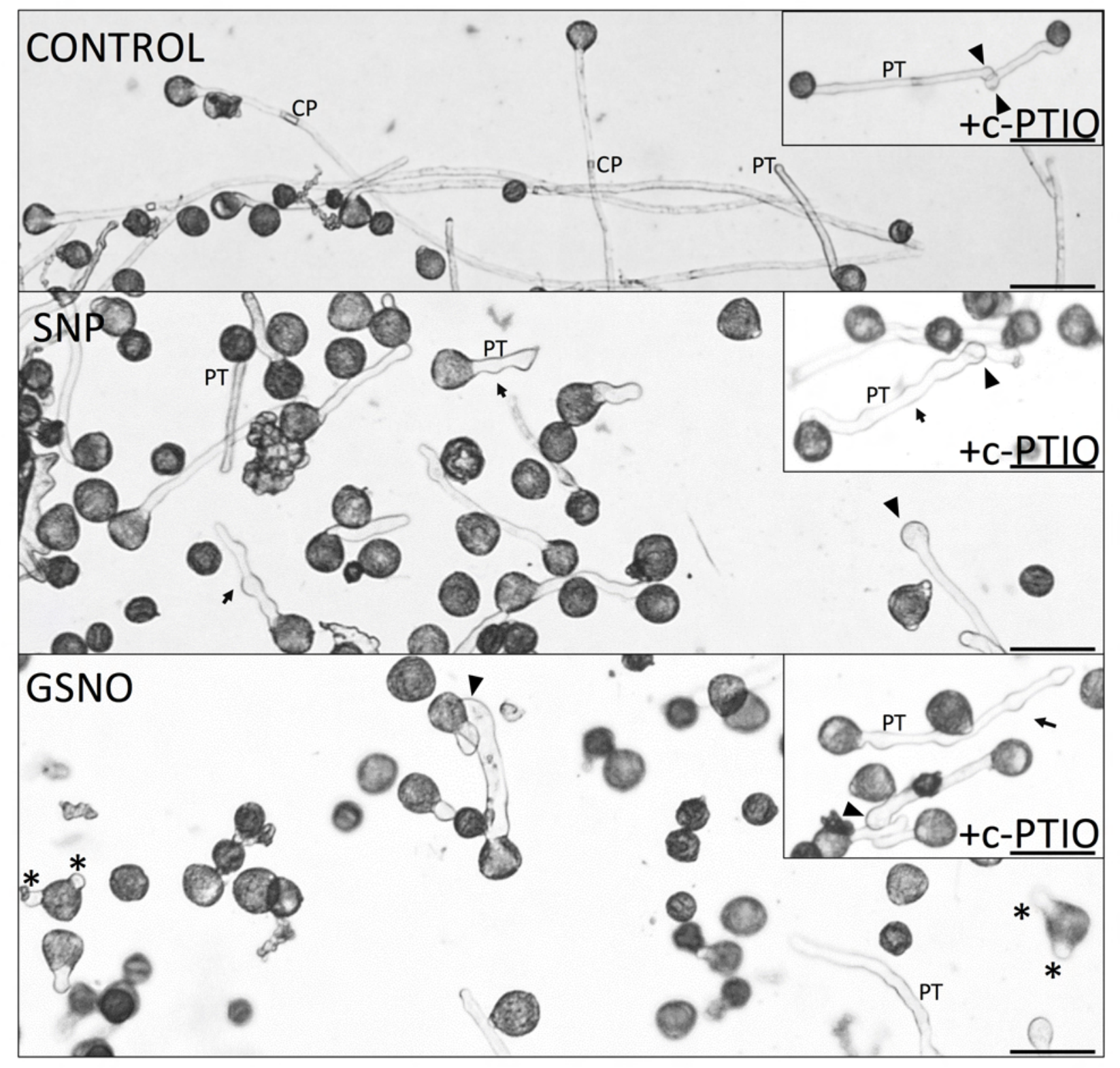




\section{ENRICHMENT ANALYSIS OF S-NITROSYLATION SITES IN OLIVE POLLEN PROTEINS}

Singular Enrichment Analysis of GO Biological Process

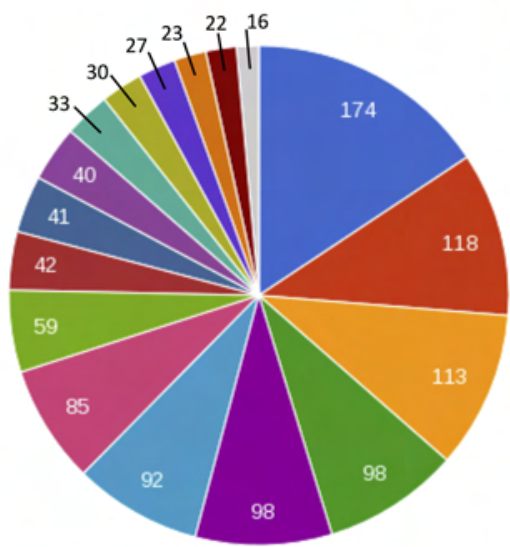

- metabolic process (BP)

response to cadmium ion (BP)

response to salt stress (BP)

embryo development ending in seed dorm ....

transport (BP)

carbohydrate metabolic process (BP)

ubiquitin-dependent protein catabolic proc.

intracellular protein transport (BP)

protein ubiquitination (BP)

pollen development (BP)

DNA repair (BP)

protein autophosphorylation (BP)

pollen tube growth (BP)

protein dephosphorylation (BP)

microtubule-based movement (BP)

pollen germination (BP)

Numerals: NUMBER OF GENES PER ANNOTATION Others

Singular Enrichment Analysis of GO Cellular Component

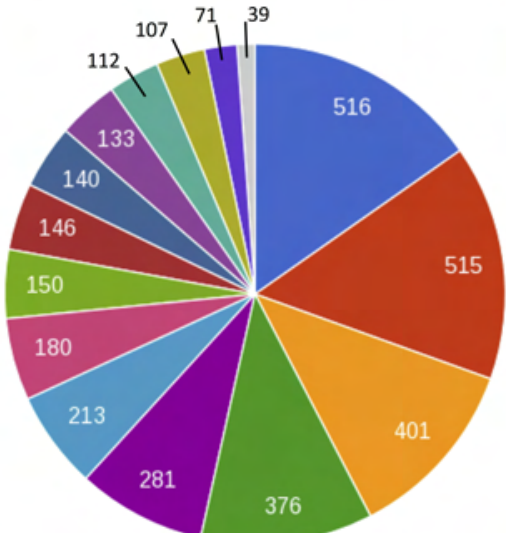

plasma membrane (CC)

nucleus (CC)

chloroplast (CC)

- membrane (CC)

- plasmodesma (CC)

mitochondrion (CC)

cytoplasm (CC)

chloroplast stroma (CC)

vacuole (CC)

- vacuolar membrane (CC)

chloroplast envelope (CC)

cell wall (CC)

endoplasmic reticulum (CC)

- Golgi apparatus (CC)

Numerals: NUMBER OF GENES PER ANNOTATION
Singular Enrichment Analysis of GO Molecular Function

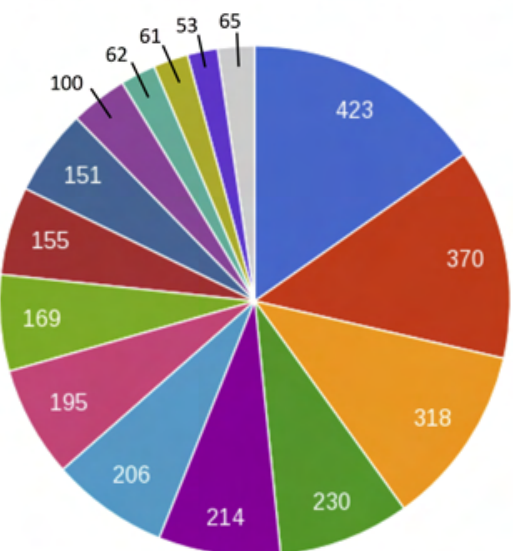

- protein binding (MF)

- ATP binding (MF)

zinc ion binding (MF)

- catalytic activity (MF)

- kinase activity (MF)

- binding (MF)

- nucleic acid binding (MF)

protein serine/threonine kinase activity (MF)

- nucleotide binding (MF)

protein kinase activity (MF)

- ubiquitin-protein ligase activity (MF)

- protein serine/threonine phosphatase

- helicase activity (MF)

- ATPase activity (MF)

Others

Numerals: NUMBER OF GENES PER ANNOTATION

Singular Enrichment Analysis of GO KEGG Patways

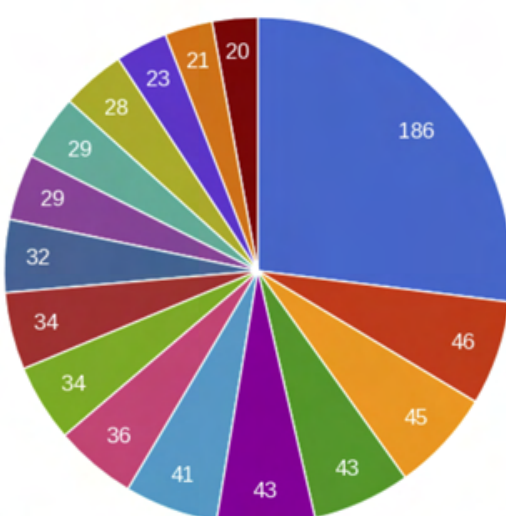

- Biosynthesis of secondary metabolites

Protein processing in endoplasmic reticulum

- Oxidative phosphorylation

Starch and sucrose metabolism

- Amino sugar and nucleotide sugar

metabolism

Glycolysis / Gluconeogenesis

Ubiquitin mediated proteolysis

- Pyruvate metabolism

- Glycerophospholipid metabolism

- Endocytosis

- mRNA surveillance pathway

Carbon fixation in photosynthetic organisms

Citrate cycle (TCA cycle)

- Phagosome

Fructose and mannose metabolism

Numerals: NUMBER OF GENES PER ANNOTATION
- Glycerolipid metabolism 


\section{ENRICHMENT ANALYSIS OF TYr-NITRATION SITES IN OLIVE POLLEN PROTEINS}

Singular Enrichment Analysis of GO Biological Process

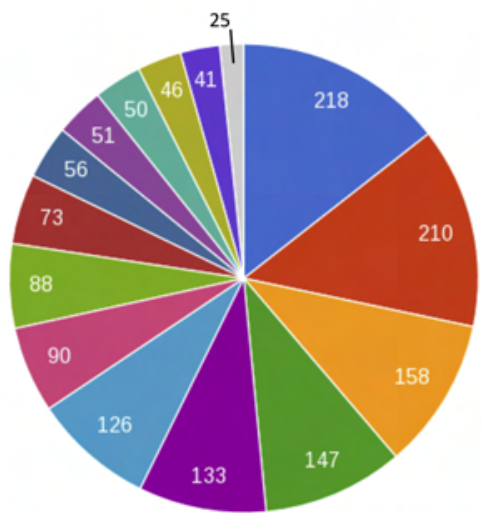

Numerals: NUMBER OF GENES PER ANNOTATION

Singular Enrichment Analysis of GO Molecular Function

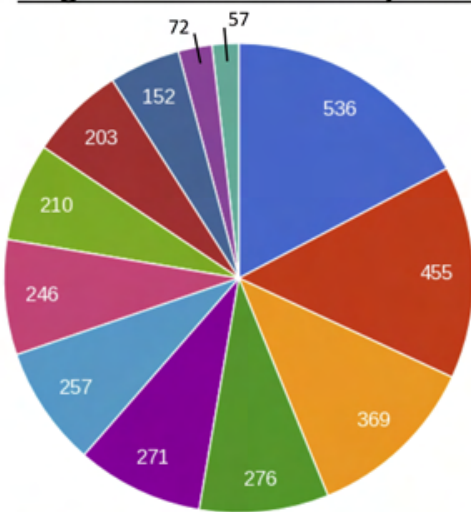

protein binding (MF)

- ATP binding (MF)

zinc ion binding (MF)

catalytic activity (MF)

- nucleic acid binding (MF)

kinase activity (MF)

binding (MF)

nucleotide binding (MF)

protein serine/threonine kinase activity (MF)

- RNA binding (MF)

- helicase activity (MF)

ATP-dependent helicase activity (MF)

Numerals: NUMBER OF GENES PER ANNOTATION

\section{Singular Enrichment Analysis of GO KEGG Patways}

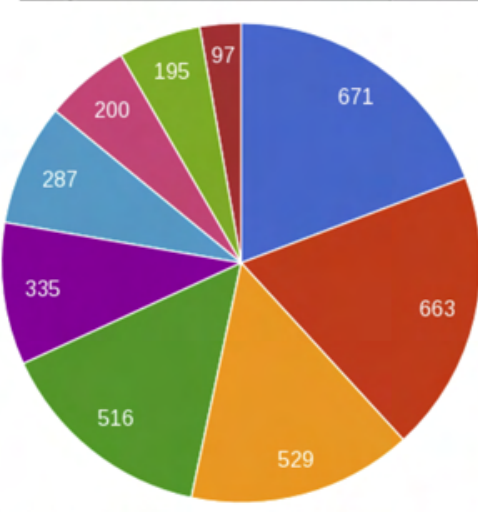

plasma membrane (CC)

nucleus (CC)

chloroplast (CC)

membrane (CC)

plasmodesma (CC)

mitochondrion (CC)

vacuole (CC)

vacuolar membrane (CC)

Golgi apparatus (CC)

Numerals: NUMBER OF GENES PER ANNOTATION

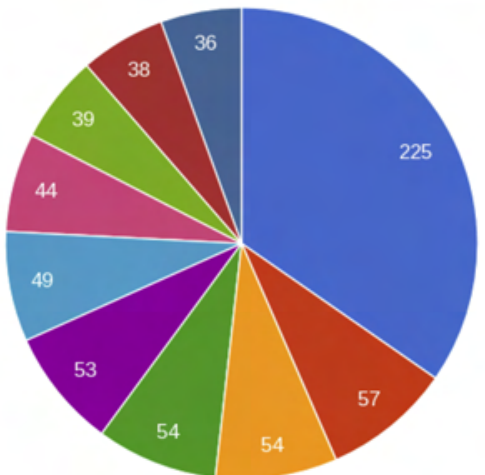

Biosynthesis of secondary metabolites

Protein processing in endoplasmic reticulum

- Spliceosome

Starch and sucrose metabolism

- Amino sugar and nucleotide sugar

etabolism

- Glycolysis / Gluconeogenesis

- Endocytosis

- Pyruvate metabolism

Citrate cycle (TCA cycle)

- Peroxisome 
iSNO-AAPair

GPS-YNO2

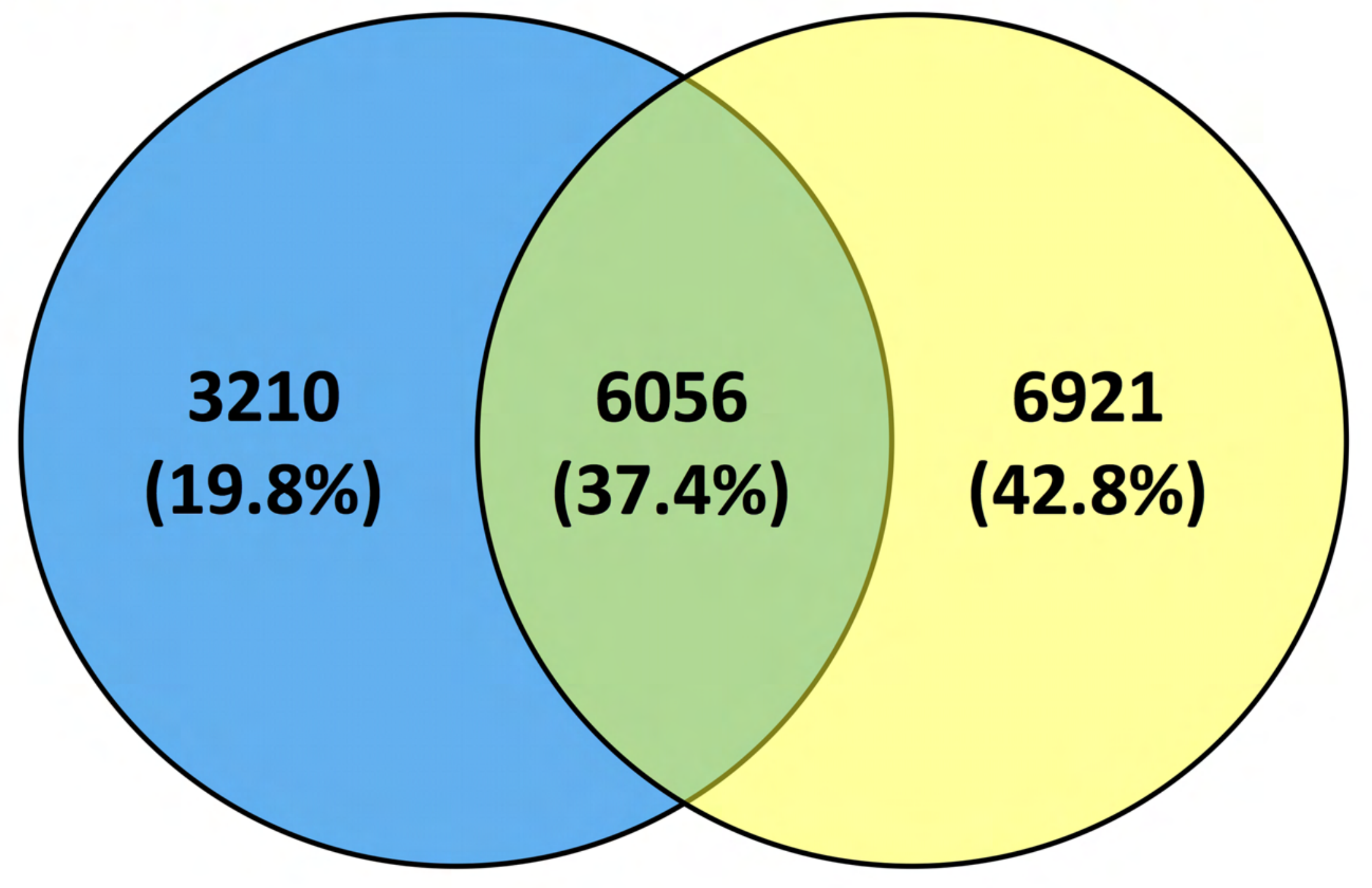




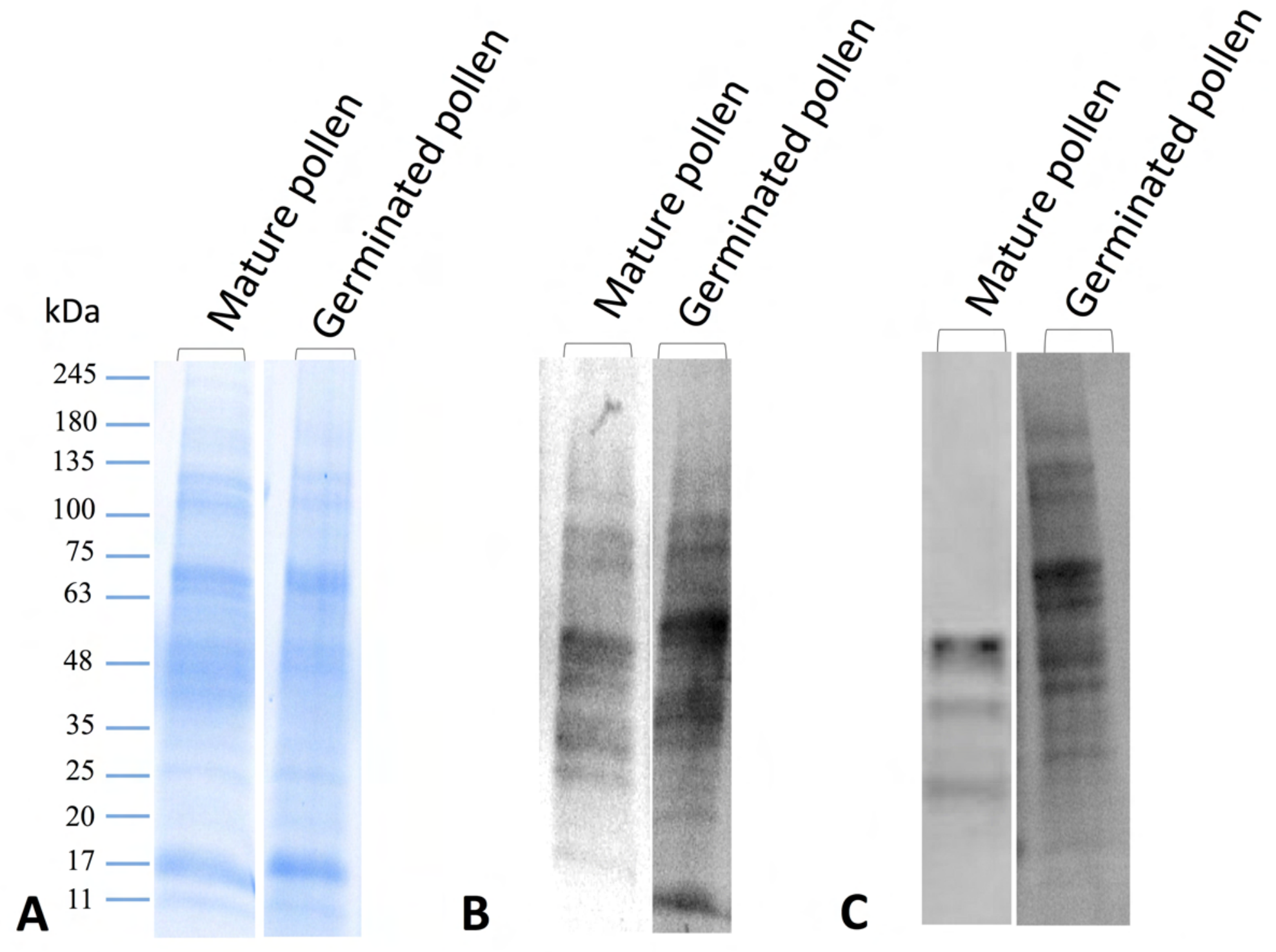




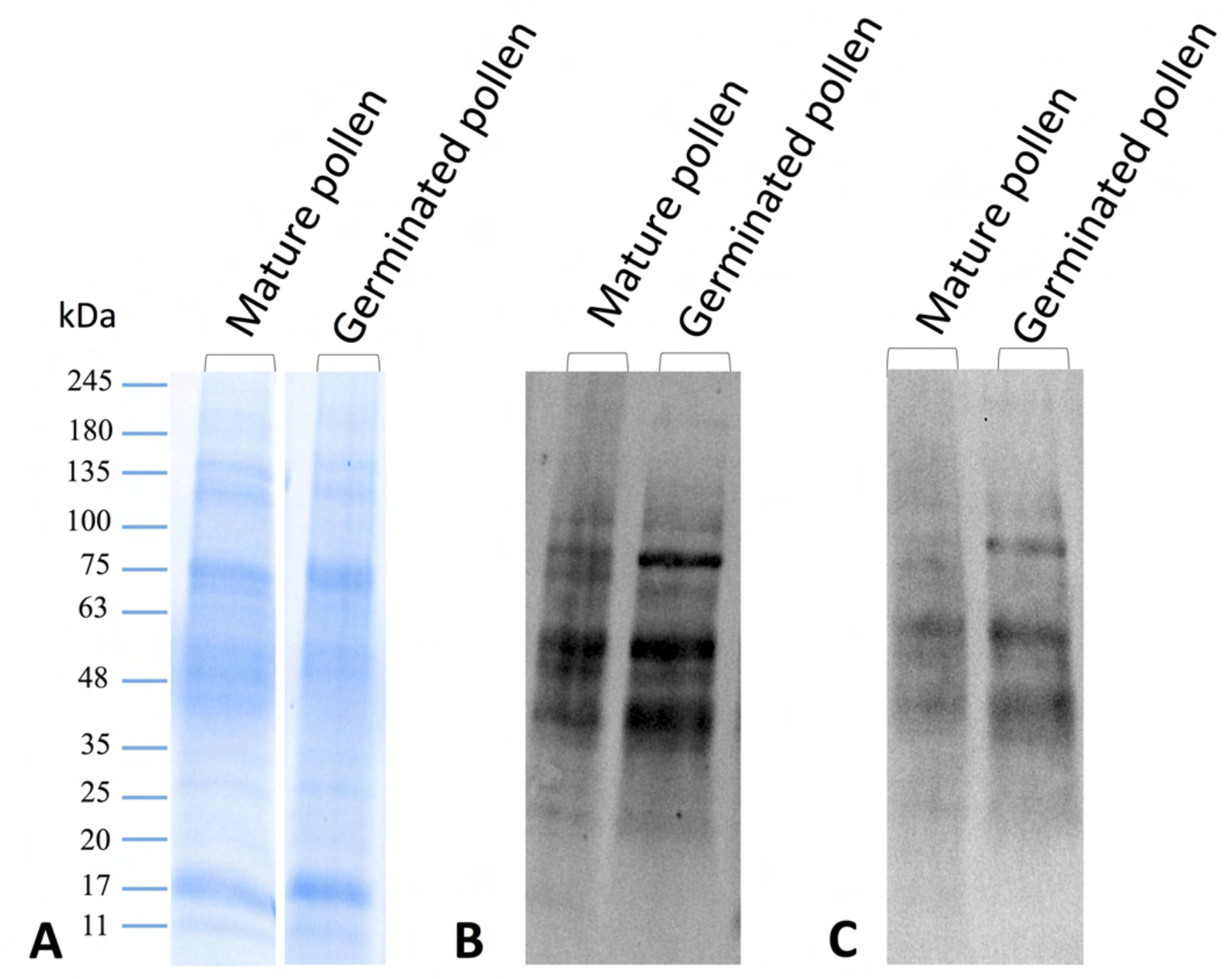




\section{iSNO-AApair SNOSite}

\section{(default) (high threshold)}

\section{GPS-SNO $\quad 9266 \quad 15285$}

(high threshold)
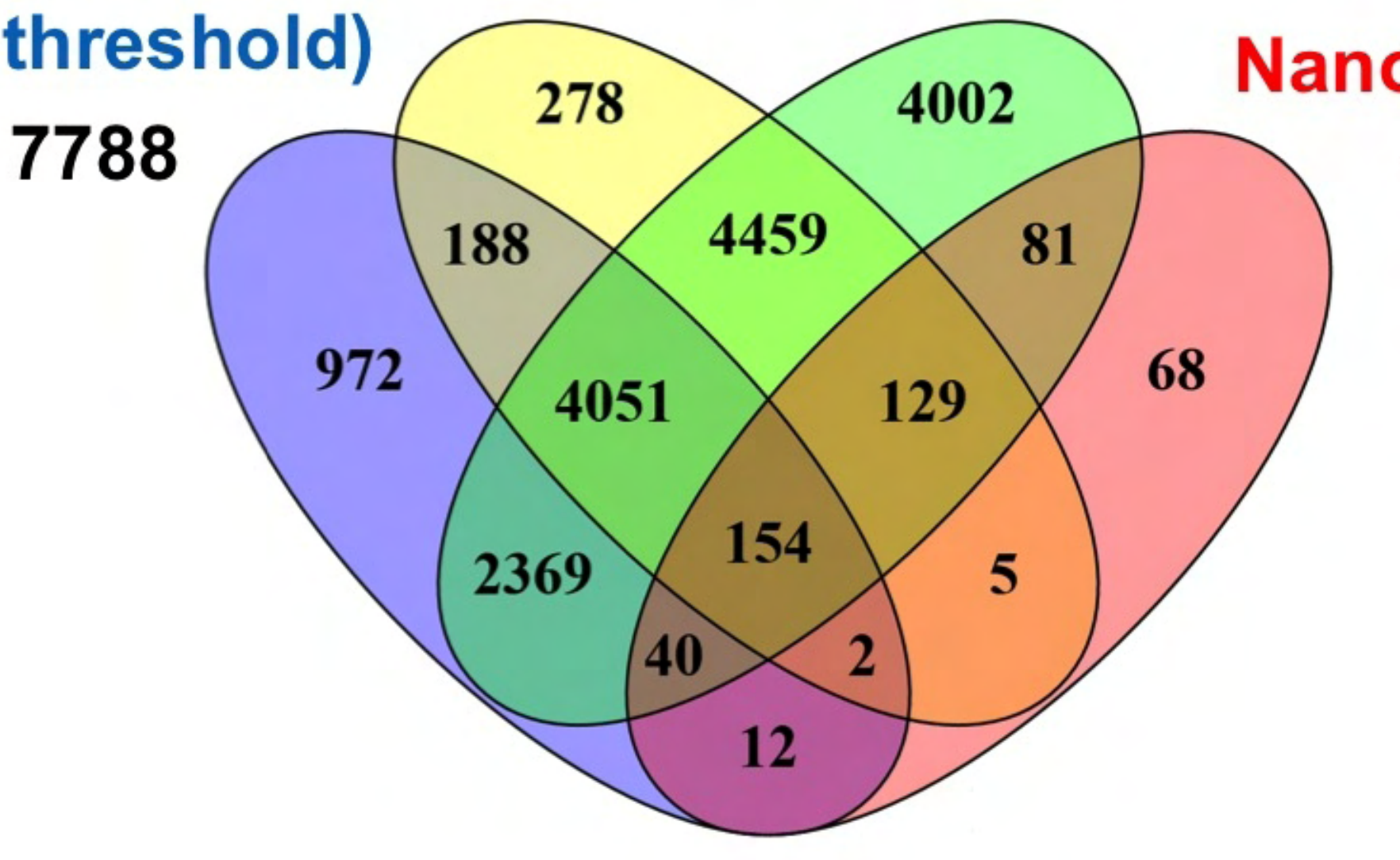

491 


\section{GPS-YNO2}

(medium -defaultthreshold)

12977

\section{(1)}

$+$

GPS-YNO2 (high threshold)

10836

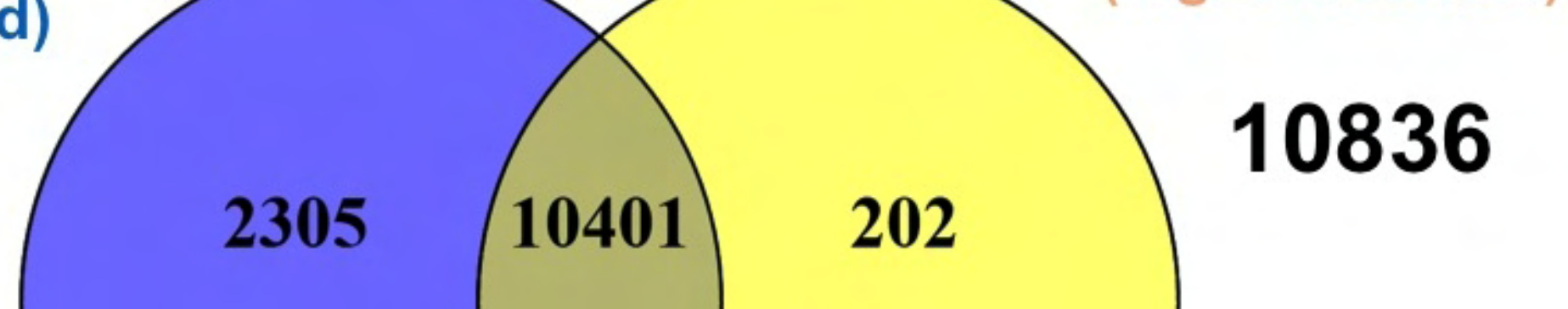




\section{HIGHLIGHTS}

- Positive correlation between NO production and pollen viability is demonstrated.

- Olive pollen emits NO/GSNO to the culture medium in a time dependent manner.

- Exogenous NO inhibits pollen germination and tube growth. Disturbed pollen tube morphology occurs after treatment with either NO donors or NO scavenger.

- Pollen predictive S-nitroso- and nitro-proteomes are highly complex and functional.

- Such NO-PTM proteins can be detected and identified by using specific antibodies. 